\title{
Liouville theorems for the Stokes equations with applications to large time estimates
}

\section{Ken Abe}

\begin{tabular}{|c|l|}
\hline Citation & Journal of Functional Analysis. 278(2); 108321 \\
\hline Issue Date & $2020-01-15$ \\
\hline Type & Journal Article \\
\hline Textversion & Author \\
\hline \multirow{3}{*}{ Rights } & $\begin{array}{l}\text { C } 2019 \text { Elsevier Inc. This manuscript version is made available under the } \\
\text { CC-BY-NC-ND 4.0 License. http://creativecommons.org/licenses/by-nc-nd/4.0/. } \\
\text { The is the accepted manuscript version. The formal published version is available at } \\
\text { https://doi.org/10.1016/j.jfa.2019.108321. }\end{array}$ \\
\hline DOI & \begin{tabular}{l}
$10.1016 / j . j f a .2019 .108321$ \\
\hline
\end{tabular} \\
\hline
\end{tabular}

\author{
Self-Archiving by Author(s) \\ Placed on: Osaka City University Repository
}




\title{
LIOUVILLE THEOREMS FOR THE STOKES EQUATIONS WITH APPLICATIONS TO LARGE TIME ESTIMATES
}

\author{
K. ABE
}

\begin{abstract}
We study Liouville theorems for the non-stationary Stokes equations in exterior domains in $\mathbb{R}^{n}$ under decay conditions for spatial variables. As applications, we prove that the Stokes semigroup is a bounded analytic semigroup on $L_{\sigma}^{\infty}$ of angle $\pi / 2$ for $n \geq 3$. We also prove large time estimates for $n=2$ with zero net force.
\end{abstract}

\section{INTRODUCTION}

We consider the Stokes equations:

$$
\begin{aligned}
& \partial_{t} v-\Delta v+\nabla q=0, \quad \operatorname{div} v=0 \quad \text { in } \Omega \times(0, \infty), \\
& v=0 \quad \text { on } \partial \Omega \times(0, \infty), \\
& v=v_{0} \quad \text { on } \Omega \times\{t=0\},
\end{aligned}
$$

for exterior domains $\Omega \subset \mathbb{R}^{n}, n \geq 2$. It is known that a solution operator (called the Stokes semigroup) $S(t): v_{0} \longmapsto v(\cdot, t)=\left(v_{i}(\cdot, t)\right)_{1 \leq i \leq n}$ forms an analytic semigroup on $L_{\sigma}^{p}$ for $p \in(1, \infty)$, of angle $\pi / 2$ [57], [31], i.e., $S(t) v_{0}$ is a holomorphic function in the half plane $\{\operatorname{Re} t>0\}$ on $L_{\sigma}^{p}$. Here, $L_{\sigma}^{p}$ denotes the $L^{p}$-closure of $C_{c, \sigma}^{\infty}$, the space of all smooth solenoidal vector fields with compact support in $\Omega$. We define $S(t)$ by the Dunford integral of the resolvent of the Stokes operator $A_{p}=\mathbb{P} \Delta$ by using the Helmholtz projection $\mathbb{P}: L^{p} \longrightarrow L_{\sigma}^{p}$ [29], [48], [56]. See, e.g., [42] for analytic semigroups.

We say that an analytic semigroup is a bounded analytic semigroup of angle $\pi / 2$ if the semigroup is bounded in the sector $\Sigma_{\theta}=\{t \in \mathbb{C} \backslash\{0\}|| \arg t \mid<\theta\}$ for each $\theta \in(0, \pi / 2)[8$, Definition 3.7.3]. The boundedness in the sector implies the bounds on the positive real line

$$
\|S(t)\| \leq C, \quad\left\|A_{p} S(t)\right\| \leq \frac{C^{\prime}}{t}, \quad t>0
$$

where $\|\cdot\|$ denotes the operator norm. The estimates (1.2) are important to study large time behavior of solutions to (1.1). In terms of the resolvent, the boundedness of $S(t)$ of angle $\pi / 2$ is equivalent to the estimate

$$
\left\|\left(\lambda-A_{p}\right)^{-1}\right\| \leq \frac{C_{\theta}}{|\lambda|}, \quad \lambda \in \Sigma_{\theta+\pi / 2}
$$

Date: August 19, 2019.

2010 Mathematics Subject Classification. 35Q35, 35K90.

Key words and phrases. Stokes equations, exterior domains, Liouville theorems. 
When $\Omega$ is a half space, $S(t)$ is a bounded analytic semigroup on $L_{\sigma}^{p}$ of angle $\pi / 2$ [47], [62], [13]. The problem becomes more difficult when $\Omega$ is an exterior domain. For $n \geq 3$, the boundedness of $S(t)$ on $L_{\sigma}^{p}$ is proved in [15] based on the resolvent estimate

$$
\left|\lambda\|\| v\left\|_{L^{p}}+|\lambda|^{1 / 2}\right\| \nabla v\left\|_{L^{p}}+\right\| \nabla^{2} v\left\|_{L^{p}} \leq C\right\| f \|_{L^{p}}, \quad 1<p<\frac{n}{2},\right.
$$

for $v=\left(\lambda-A_{p}\right)^{-1} f$ and $\lambda \in \Sigma_{\theta+\pi / 2} \cup\{0\}$. The estimate (1.4) implies (1.3) for $p \in(1, n / 2)$ and the case $p \in[n / 2, \infty)$ follows from a duality. Due to the restriction on $p$, the twodimensional case is more involved. Indeed, (1.4) is optimal in the sense that

$$
\left\|\nabla^{2} v\right\|_{L^{p}} \leq C\left\|A_{p} v\right\|_{L^{p}}, \quad v \in D\left(A_{p}\right)
$$

is not valid for any $p \in[n / 2, \infty)$ [14]. Here, $D\left(A_{p}\right)=W^{2, p} \cap W_{0}^{1, p} \cap L_{\sigma}^{p}$ and $W_{0}^{1, p}$ denotes the space of all $f \in W^{1, p}$ vanishing on $\partial \Omega$. For $n=2$, the boundedness of the Stokes semigroup on $L_{\sigma}^{p}$ is proved in [17] based on layer potentials for the Stokes resolvent (see also [63]).

We study the case $p=\infty$. When $\Omega$ is a half space, $S(t)$ is a bounded analytic semigroup on $L_{\sigma}^{\infty}$ of angle $\pi / 2$ by explicit solution formulas [27], [58]. Here, $L_{\sigma}^{\infty}$ is defined by

$$
L_{\sigma}^{\infty}(\Omega)=\left\{f \in L^{\infty}(\Omega) \mid \int_{\Omega} f \cdot \nabla \Phi \mathrm{d} x=0, \nabla \Phi \in G^{1}(\Omega)\right\}
$$

and $G^{1}(\Omega)=\left\{\nabla \Phi \in L^{1}(\Omega) \mid \Phi \in L_{\text {loc }}^{1}(\Omega)\right\}$. For a half space and domains with compact boundary, $L_{\sigma}^{\infty}$ agrees with the space of all $f \in L^{\infty}$ satisfying $\operatorname{div} f=0$ in $\Omega$ and $f \cdot N=0$ on $\partial \Omega$. Here, $N$ is the unit outward normal vector field on $\partial \Omega$. Since $S(t)$ is bounded on $L^{\infty}$, the associated generator $A_{\infty}$ is defined also for $p=\infty$. For bounded domains [3] and exterior domains [4], analyticity of the semigroup on $L_{\sigma}^{\infty}$ follows from the a priori estimate

$$
\|v\|_{L^{\infty}}+t^{1 / 2}\|\nabla v\|_{L^{\infty}}+t\left\|\nabla^{2} v\right\|_{L^{\infty}}+t\left\|\partial_{t} v\right\|_{L^{\infty}}+t\|\nabla q\|_{L^{\infty}} \leq C\left\|v_{0}\right\|_{L^{\infty}}
$$

for $v=S(t) v_{0}$ and $t \leq T$. The estimate (1.5) implies (1.2) for $t \leq T$ and that $S(t)$ is analytic on $L_{\sigma}^{\infty}$. Moreover, the angle of analyticity is $\pi / 2$ by the resolvent estimate on $L_{\sigma}^{\infty}$ [5]. When $\Omega$ is bounded, the sup-norms in (1.5) exponentially decay as $t \rightarrow \infty$ and $S(t)$ is a bounded analytic semigroup on $L_{\sigma}^{\infty}$ of angle $\pi / 2$. For exterior domains, it is non-trivial whether the Stokes semigroup is a bounded analytic semigroup on $L_{\sigma}^{\infty}$.

For the Laplace operator or general elliptic operators, it is known that corresponding semigroups are analytic on $L^{\infty}$ of angle $\pi / 2$ [46], [61], [42]. Moreover, if the operators are uniformly elliptic, by Gaussian upper bounds for complex time heat kernels, the semigroups are bounded analytic on $L^{\infty}$ of angle $\pi / 2$. See [26, Chapter 3]. In particular, the heat semigroup with the Dirichlet boundary condition in an exterior domain for $n \geq 2$ is a bounded analytic semigroup on $L^{\infty}$ of angle $\pi / 2$. For the Stokes equations, the Gaussian upper bound may not hold. See [27], [58], [51] for a half space.

Large time $L^{\infty}$-estimates of the Stokes semigroup have been studied for $n \geq 3$. Maremonti [43] proved the estimate 


$$
\left\|S(t) v_{0}\right\|_{L^{\infty}} \leq C\left\|v_{0}\right\|_{L^{\infty}}, \quad t>0
$$

for exterior domains and $n \geq 3$ based on the short time estimate in [3]. Subsequently, HieberMaremonti [36] proved the estimate $t\left\|A S(t) v_{0}\right\|_{L^{\infty}} \leq C\left\|v_{0}\right\|_{L^{\infty}}$ for $t>0$ and the results are extended in [12] for complex time $t \in \Sigma_{\theta}$ and $\theta \in(0, \pi / 2)$ based on the approach in [43]. Of these papers, the case $n=2$ is excluded. We are able to observe the difference between $n \geq 3$ and $n=2$ from the representation formula of the Stokes flow due to Mizumachi [49], [35]. See below (1.9). In this paper, we study large time behavior of Stokes flows for $n \geq 2$ by a different approach.

Our approach is by a Liouville theorem. A Liouville theorem is a fundamental property to study regularity problems. It rules out non-trivial solutions defined in $\Omega \times(-\infty, 0]$, called ancient solutions. See [40], [54] for Liouville theorems of the Navier-Stokes equations and [39] for the Stokes equations. Liouville theorems are also important to study large time behavior of solutions. In this paper, we prove non-existence of ancient solutions of (1.1) in exterior domains under spatial decay conditions. We then apply our Liouville theorems and prove the large time estimate (1.6) for complex time $t \in \Sigma_{\theta}$ and $\theta \in(0, \pi / 2)$.

We say that $v \in L_{\mathrm{loc}}^{1}(\bar{\Omega} \times(-\infty, 0])$ is an ancient solution to the Stokes equations (1.1) if $\operatorname{div} v=0$ in $\Omega \times(-\infty, 0), v \cdot N=0$ on $\partial \Omega \times(-\infty, 0)$ and

$$
\int_{-\infty}^{0} \int_{\Omega} v \cdot\left(\partial_{t} \varphi+\Delta \varphi\right) \mathrm{d} x \mathrm{~d} t=0
$$

for all $\varphi \in C_{c}^{2,1}(\bar{\Omega} \times(-\infty, 0])$ satisfying $\operatorname{div} \varphi=0$ in $\Omega \times(-\infty, 0)$ and $\varphi=0$ on $\partial \Omega \times(-\infty, 0) \cup$ $\Omega \times\{t=0\}$. The conditions $\operatorname{div} v=0$ and $v \cdot N=0$ are understood in the sense that

$$
\int_{\Omega} v \cdot \nabla \Phi \mathrm{d} x=0, \quad \text { a.e. } t \in(-\infty, 0)
$$

for all $\Phi \in C_{c}^{1}(\bar{\Omega})$. Our first result is:

Theorem 1.1 (Liouville theorem). Let $\Omega$ be an exterior domain with $C^{3}$-boundary in $\mathbb{R}^{n}$, $n \geq 2$. Let $v$ be an ancient solution to the Stokes equations (1.1). Assume that

$$
v \in L^{\infty}\left(-\infty, 0 ; L^{p}\right) \quad \text { for } p \in(1, \infty) \text {. }
$$

Then, $v \equiv 0$.

If one removes the spatial decay condition (1.8), the assertion of Theorem 1.1 becomes false for $n \geq 3$ due to existence of stationary solutions which are asymptotically constant as $|x| \rightarrow \infty$ [14]. See Remarks 2.7. For $n=2$, it is known that bounded stationary solutions do not exist [22]. We show that ancient solutions in $L^{\infty}\left(-\infty, 0 ; L^{p}\right)$ are extendable to bounded entire functions by using boundedness and analyticity of the Stokes semigroup. 
The Liouville theorem then follows from the kernel property of the Stokes operator on $L_{\sigma}^{p}$, i.e., $\mathcal{N}\left(A_{p}\right)=\left\{v \in D\left(A_{p}\right) \mid A_{p} v=0\right\}=\{0\}$. See [33] for $n \geq 3$ and [22], [17] for $n=2$.

Theorem 1.1 is useful to study the large time estimate (1.6) for $t>0$. We invoke the representation formula of the Stokes flow

$$
v(x, t)=\int_{\Omega} \Gamma(x-y, t) v_{0}(y) \mathrm{d} y+\int_{0}^{t} \int_{\partial \Omega} V(x-y, t-s) T(y, s) N(y) \mathrm{d} H(y) \mathrm{d} s .
$$

Here, $T=\nabla v+\nabla^{T} v-q I$ is the stress tensor and $V=\left(V_{i j}(x, t)\right)_{1 \leq i, j \leq n}$ is the Oseen tensor

$$
V_{i j}(x, t)=\Gamma(x, t) \delta_{i j}+\partial_{i} \partial_{j} \int_{\mathbb{R}^{n}} E(x-y) \Gamma(y, t) \mathrm{d} y,
$$

defined by the heat kernel $\Gamma(x, t)=(4 \pi t)^{-n / 2} e^{-|x|^{2} / 4 t}$ and the fundamental solutions of the Laplace equation $E$, i.e., $E(x)=(n(n-2) \alpha(n))^{-1}|x|^{-(n-2)}$ for $n \geq 3$ and $E(x)=-(2 \pi)^{-1} \log |x|$ for $n=2$, where $\alpha(n)$ denotes the volume of the unit ball in $\mathbb{R}^{n}$. The formula (1.9) is obtained by regarding $v=S(t) v_{0}$ as the Stokes flow in $\mathbb{R}^{n}$ with a measure as the external force. See Lemma 3.8. It describes the asymptotic behavior of bounded Stokes flows as $|x| \rightarrow \infty$. We show that if the Stokes flow is bounded for all $t>0$, the stress tensor is also bounded on $\partial \Omega$. Observe that by the pointwise estimate of the Oseen tensor

$$
|V(x, t)| \leq \frac{C}{\left(|x|+t^{1 / 2}\right)^{n}}, \quad x \in \mathbb{R}^{n}, t>0,
$$

the remainder term is estimated by

$$
\left|v(x, t)-\int_{\Omega} \Gamma(x-y, t) v_{0}(y) \mathrm{d} y\right| \leq \frac{C}{|x|^{n-2}} \sup _{0<s \leq t}\|T\|_{L^{\infty}(\partial \Omega)}(s),
$$

for $|x| \geq 2 R_{0}$ and $t>0$ such that $\Omega^{c} \subset B_{0}\left(R_{0}\right)$, where $B_{0}\left(R_{0}\right)$ denotes the open ball centered at the origin with radius $R_{0}>0$. The right-hand side is decaying as $|x| \rightarrow \infty$ uniformly for $t>0$ if $n \geq 3$. We show that the large time estimate (1.6) is reduced to showing nonexistence of ancient solutions by a contradiction argument. Since (1.12) yields a spatial decay condition for ancient solutions as $|x| \rightarrow \infty$, we are able to derive a contradiction by applying the Liouville theorem (Theorem 1.1).

We apply a similar argument on the half line $\gamma=\{t \in \mathbb{C} \backslash\{0\} \mid \arg t=\theta\}$ and prove (1.6) for complex time $t \in \Sigma_{\theta}$ and $\theta \in(0, \pi / 2)$. To this end, we consider ancient solutions in the sector

$$
\Lambda=\{t \in \mathbb{C} \backslash\{0\} \mid-\pi+\theta \leq \arg t \leq-\pi / 2\} .
$$

We set the segment $I_{T}=\{t \in \Lambda \mid-T \leq \operatorname{Re} t \leq 0, \operatorname{Im} t=-T \tan \theta\}$ for $T>0$. We say that $v$ is an ancient solution in $\Omega \times \Lambda$ if $v$ satisfies the Stokes equations (1.1) on each segment $I_{T}$ for $T>0$ in a weak sense, i.e., $v \in L_{\mathrm{loc}}^{1}\left(\bar{\Omega} \times I_{T}\right)$ satisfies $\operatorname{div} v=0$ in $\Omega \times I_{T}, v \cdot N=0$ on $\partial \Omega \times I_{T}$ and 


$$
\int_{-T}^{0} \int_{\Omega} v(x, \alpha+i \beta)\left(\partial_{\alpha} \varphi+\Delta \varphi\right) \mathrm{d} x \mathrm{~d} \alpha=-\int_{\Omega} v(x,-T+i \beta) \varphi(x,-T) \mathrm{d} x,
$$

for $\beta=-T \tan \theta$ and all $\varphi \in C_{c}^{2,1}(\bar{\Omega} \times[-T, 0])$ satisfying $\operatorname{div} \varphi=0$ in $\Omega \times(-T, 0)$ and $\varphi=0$ on $\partial \Omega \times(-T, 0) \cup \Omega \times\{\alpha=0\}$. We prove non-existence of ancient solutions in the sector $\Lambda$ under the condition $v \in L^{\infty}\left(\Lambda ; L^{p}\right)$ for $p \in(1, \infty)$ (Theorem 2.6). Since the formula (1.9) is extendable for complex time, we apply the Liouville theorem in the sector and obtain (1.6) for $t \in \Sigma_{\theta}$ and $\theta \in(0, \pi / 2)$. We now state our main results.

Theorem 1.2. When $n \geq 3$, the Stokes semigroup is a bounded analytic semigroup on $L_{\sigma}^{\infty}$ of angle $\pi / 2$.

For $n=2$, the remainder term estimate (1.12) is different. By a simple calculation from the formula (1.9), we see an asymptotic profile of the two-dimensional Stokes flow:

$$
\left|v(x, t)-\int_{\Omega} \Gamma(x-y, t) v_{0}(y) \mathrm{d} y-\int_{0}^{t} V(x, t-s) F(s) \mathrm{d} s\right| \leq \frac{C}{|x|} \sup _{0<s \leq t}\|T\|_{L^{\infty}(\partial \Omega)}(s),
$$

for $|x| \geq 2 R_{0}$ and $t>0$, with the net force

$$
F(s)=\int_{\partial \Omega} T(y, s) N(y) \mathrm{d} H(y)
$$

Since $\left|\int_{0}^{t} V(x, s) \mathrm{d} s\right| \lesssim \log \left(1+t /|x|^{2}\right)$ by (1.11), the decay as $|x| \rightarrow \infty$ of the third term in (1.14) is not uniform for $t>0$ in contrast to (1.12) for $n \geq 3$. If the net force vanishes, the situation is the same as $n=3$ and we are able to prove (1.6). For example, if initial data is rotationally symmetric, the net force vanishes. Following [18], we consider initial data invariant under a cyclic group or a dihedral group. For integers $m \geq 2$, we set the matrices

$$
R_{m}=\left(\begin{array}{cc}
\cos (2 \pi / m) & -\sin (2 \pi / m) \\
\sin (2 \pi / m) & \cos (2 \pi / m)
\end{array}\right), \quad J=\left(\begin{array}{cc}
1 & 0 \\
0 & -1
\end{array}\right)
$$

Let $C_{m}$ denote the cyclic group of order $m$ generated by the rotation $R_{m}$. Let $D_{m}$ denote the dihedral group of order $2 m$ generated by $R_{m}$ and the reflection $J$. Any finite subgroup of the orthogonal group $O(2)$ is either a cyclic group or a dihedral group. See [34, Chapter 2]. Let $G$ be a subgroup of $O(2)$ and $\Omega^{c}$ be a disk centered at the origin. We say that a vector field $v$ is $G$-covariant if $v(x)={ }^{t} A v(A x)$ for all $A \in G$ and $x \in \Omega$. It is known that if $v_{0}$ is $C_{m}$-covariant, so is $v=S(t) v_{0}$ and the net force vanishes, i.e., $F \equiv 0$ [35]. Thus for $C_{m}$-covariant vector fields $v_{0} \in L_{\sigma}^{\infty}$, the remainder term estimate is the same as $n=3$.

Theorem 1.3. For $n=2$, the estimate (1.6) holds for $t \in \Sigma_{\theta}$ and $v_{0} \in L_{\sigma}^{\infty}$, for which the net force vanishes (e.g., $C_{m}$-covariant vector fields when $\Omega^{c}$ is a disk.) 
Theorem 1.3 improves the pointwise estimates of the two-dimensional Navier-Stokes flows for rotationally symmetric initial data [35], in which (1.6) is noted as an open question together with the applications to the nonlinear problem. We are able to apply (1.6) to improve the results although initial data is restricted to rotationally symmetric. See Remarks 5.4 (iii).

We hope it is possible to extend our approach to study the case with net force, for which (1.6) is unknown even if initial data is with finite Dirichlet integral. The estimate (1.6) with non-vanishing net force is important to study large time behavior of asymptotically constant solutions as $|x| \rightarrow \infty$. We refer to [2] for asymptotically constant solutions of the twodimensional Navier-Stokes equations. See also [44].

This paper is organized as follows. In Section 2, we prove Theorem 1.1. In Section 3, we give a spatial decay estimate of $S(t) v_{0}$ to prove (1.6) by a contradiction argument. In Section 4, we prove (1.6) for positive time. In Section 5, we prove (1.6) for complex time (Theorems 1.2). After the proof of Theorem 1.2, we note the case $n=2$ (Theorem 1.3).

\section{LIOUVILLE THEOREMS ON $L^{p}$}

We prove Theorem 1.1. We represent ancient solutions $v \in L^{\infty}\left(-\infty, 0 ; L^{p}\right)$ as $v(t)=$ $S(t+T) v(-T)$ for $T>0$ by the Stokes semigroup $S(t)$. Since $S(t)$ is analytic and bounded in the sector $\Sigma_{\theta}$ for $\theta \in(0, \pi / 2)$, ancient solutions are bounded entire functions on $L^{p}$, i.e., $\partial_{t} v \equiv 0$. Theorem 1.1 then easily follows from the kernel property of the Stokes operator $\mathcal{N}\left(A_{p}\right)=\left\{v \in D\left(A_{p}\right) \mid A_{p} v=0\right\}=\{0\}$.

2.1. An adjoint problem. To represent ancient solutions by $S(t)$, we prove a uniqueness theorem for the Stokes equations (1.1) (Lemma 2.5). We apply a duality argument and reduce the uniqueness to existence of solutions to an adjoint problem (Lemma 2.3). To do this, we recall the Helmholtz projection and the Stokes operator on $L^{p}$.

Proposition 2.1. (i) $\operatorname{Let} C_{c, \sigma}^{\infty}(\Omega)=\left\{v \in C_{c}^{\infty}(\Omega) \mid \operatorname{div} v=0\right\}$,

$$
L_{\sigma}^{p}(\Omega)={\overline{C_{c, \sigma}^{\infty}(\Omega)}}^{\|\cdot\|_{L^{p}}}, \quad G^{p}(\Omega)=\left\{\nabla \Phi \in L^{p}(\Omega) \mid \Phi \in L_{l o c}^{p}(\Omega)\right\}, \quad p \in(1, \infty) .
$$

For $f \in L^{p}$, there exists a unique $f_{0} \in L_{\sigma}^{p}$ and $\nabla \Phi \in L^{p}$ such that $f=f_{0}+\nabla \Phi$ and

$$
\left\|f_{0}\right\|_{L^{p}}+\|\nabla \Phi\|_{L^{p}} \leq C\|f\|_{L^{p}}
$$

with some constant $C$, independent of $f$. We call $\mathbb{P}: f \longmapsto f_{0}$ the Helmholtz projection operator. Moreover, we have

$$
L_{\sigma}^{p}(\Omega)=\left\{f \in L^{p}(\Omega) \mid \int_{\Omega} f \cdot \nabla \Phi d x=0, \nabla \Phi \in G^{p^{\prime}}(\Omega)\right\}, \quad 1 / p+1 / p^{\prime}=1
$$


The space $G_{0}(\Omega)=\left\{\nabla \Phi \mid \Phi \in C_{c}^{\infty}(\bar{\Omega})\right\}$ is dense in $G^{p}(\Omega)$ with respect to $\|\nabla \cdot\|_{L^{p}}$. (ii) Set

$$
\begin{aligned}
& A_{p} f=\mathbb{P} \Delta f, \quad f \in D\left(A_{p}\right), \\
& D\left(A_{p}\right)=W^{2, p} \cap W_{0}^{1, p} \cap L_{\sigma}^{p}(\Omega),
\end{aligned}
$$

with the graph-norm $\|v\|_{D\left(A_{p}\right)}=\|v\|_{L^{p}}+\left\|A_{p} v\right\|_{L^{p}}$. Then, $D\left(A_{p}\right) \subset W^{2, p}$ with continuous injection.

(iii) The Stokes operator $A_{p}$ generates a bounded $C_{0}$-analytic semigroup $S(t)=e^{t A_{p}}$ on $L_{\sigma}^{p}$ of angle $\pi / 2$. Moreover, $\mathcal{N}\left(A_{p}\right)=\left\{v \in D\left(A_{p}\right) \mid A_{p} v=0\right\}=\{0\}$ and $R\left(A_{p}\right)=\left\{A_{p} v \mid v \in D\left(A_{p}\right)\right\}$ is dense in $L_{\sigma}^{p}$. In particular, we have

$$
\lim _{t \rightarrow \infty}\left\|S(t) v_{0}\right\|_{L^{p}}=0, \quad v_{0} \in L_{\sigma}^{p}(\Omega)
$$

Proof. The assertions (i) and (ii) are proved in [56, Theorem 1.4, Lemma 3.7] and [19]. The boundedness of $S(t)$ is proved in [15] for $n \geq 3$ and [17] for $n=2$. The properties of the kernel and the range are proved in [33, Corollary 3.6 (i)] for $n \geq 3$ and [22], [17, p.297] for $n=2$. The decay (2.3) follows from the density of $R\left(A_{p}\right)$ in $L_{\sigma}^{p}$.

Proposition 2.2. For $g_{0} \in C_{c}^{\infty}(\Omega \times(0, T))$ satisfying div $g_{0}=0$, there exists a solution $(\psi, \nabla s) \in C^{2,1}(\bar{\Omega} \times[0, T]) \times C(\bar{\Omega} \times[0, T])$ of

$$
\begin{array}{r}
\partial_{t} \psi-\Delta \psi+\nabla s=g_{0}, \quad \operatorname{div} \psi=0 \quad \text { in } \Omega \times(0, T), \\
\psi=0 \quad \text { on } \partial \Omega \times(0, T) \cup \Omega \times\{t=0\},
\end{array}
$$

such that $\partial_{t}^{s} \partial_{x}^{k} \psi, \nabla s \in L^{\infty}\left(0, T ; L^{q}\right)$ for $2 s+|k| \leq 2$ and $q \in(1, \infty)$.

Proof. We set

$$
\psi(x, t)=\int_{0}^{t} S(t-s) g_{0}(s) \mathrm{d} s, \quad \nabla s=(1-\mathbb{P}) \Delta \psi
$$

Since $g_{0}$ is smooth and $D\left(A_{q}\right) \subset W^{2, q}$ by Proposition 2.1 (ii), $\partial_{t}^{s} \partial_{x}^{k} \psi, \nabla s \in L^{\infty}\left(0, T ; L^{q}\right)$ for $2 s+|k| \leq 2$. Since $\partial_{t} \psi-A_{q} \psi=g_{0}$ on $L^{q},(\psi, \pi)$ satisfies (2.4).

It remains to show that $(\psi, s)$ is continuous up to second orders in $\bar{\Omega} \times[0, T]$. Since $g$ is smooth, in particular $A_{q} \psi \in C^{1}\left([0, T] ; L^{q}\right) \cap C\left([0, T] ; W^{1, q}\right)$. We take bounded domains $\Omega^{\prime \prime}$ and $\Omega^{\prime}$ such that $\Omega^{\prime \prime} \subset \Omega^{\prime} \subset \Omega$. Since the boundary is $C^{3}$, we apply the higher regularity estimate for the Stokes operator [30, Theorem IV.5.1] to estimate

$$
\|\psi\|_{W^{3, q}\left(\Omega^{\prime \prime}\right)}+\|s\|_{W^{2, q\left(\Omega^{\prime \prime}\right)}} \leq C\left(\left\|A_{q} \psi\right\|_{W^{1, q}\left(\Omega^{\prime}\right)}+\|\psi\|_{W^{1, q}\left(\Omega^{\prime}\right)}+\|s\|_{L^{q}\left(\Omega^{\prime}\right)}\right) .
$$


Thus, $\psi \in C\left([0, T] ; W_{\text {loc }}^{3, q}(\bar{\Omega})\right)$. By the Sobolev embedding for $q>n$, we see that $\nabla^{2} \psi$ is continuous in $\bar{\Omega} \times[0, T]$. Since $A_{q} \partial_{t} \psi \in C\left([0, T] ; L^{q}\right), \partial_{t} \psi$ is continuous in $\bar{\Omega} \times[0, T]$. By (2.4), $\nabla s$ is continuous in $\bar{\Omega} \times[0, T]$. This completes the proof

Lemma 2.3. For $f_{0} \in C_{c}^{\infty}(\Omega \times(0, T))$ satisfying div $f_{0}=0$, there exists a solution $(\varphi, \nabla \pi) \in$ $C^{2,1}(\bar{\Omega} \times[0, T]) \times C(\bar{\Omega} \times[0, T])$ of

$$
\begin{array}{r}
\partial_{t} \varphi+\Delta \varphi-\nabla \pi=f_{0}, \quad \operatorname{div} \varphi=0 \quad \text { in } \Omega \times(0, T), \\
\varphi=0 \quad \text { on } \partial \Omega \times(0, T) \cup \Omega \times\{t=T\},
\end{array}
$$

such that $\partial_{t}^{s} \partial_{x}^{k} \varphi, \nabla \pi \in L^{\infty}\left(0, T ; L^{q}\right)$ for $2 s+|k| \leq 2$ and $q \in(1, \infty)$.

Proof. For $g(x, t)=-f(x, T-t)$, we take $(\psi, s)$ by Proposition 2.2 and set $\varphi(x, t)=\psi(x, T-t)$, $\pi(x, t)=s(x, T-t)$. Then, $(\varphi, \pi)$ satisfies the desired properties.

2.2. Uniqueness. We apply Lemma 2.3 to prove the uniqueness (Lemma 2.5). For the proof, we use the Bogovskiî operator.

Proposition 2.4. (i) Let $D=\left\{x \in \mathbb{R}^{n}|1<| x \mid<2\right\}$ and $L_{a v}^{q}(D)=\left\{h \in L^{q}(D) \mid \int_{D} h d x=0\right\}$. There exists a bounded linear operator $B: L_{a v}^{q}(D) \longrightarrow W_{0}^{1, q}(D), q \in(1, \infty)$, such that $w=B(h)$ satisfies

$$
\operatorname{div} w=h \quad \text { in } D, \quad w=0 \quad \text { on } \partial D
$$

Moreover, the operator B acts as a bounded operator from $W_{0}^{k, p}(D)$ to $W_{0}^{k+1, q}(D)$ for positive integers $k$. Here, $W_{0}^{k, q}(D)$ denotes the $W^{k, q}$-closure of $C_{c}^{\infty}(D)$.

(ii) Let $D_{R}=\{R<|x|<2 R\}$. There exists a bounded operator $B_{R}: L_{a v}^{q}\left(D_{R}\right) \longrightarrow W_{0}^{1, q}\left(D_{R}\right)$ satisfying (2.6) in $D_{R}$. Moreover, the estimate

$$
\left\|\nabla^{k+1} B_{R}(h)\right\|_{L^{q}\left(D_{R}\right)} \leq C\left\|\nabla^{k} h\right\|_{L^{q}\left(D_{R}\right)}
$$

holds with some constant $C$, independent of $R>0$.

Proof. See [10], [16], [30, Theorem III.3.3] for the assertion (i). The operator $B_{R}$ is constructed by (i) and dilation. 
Lemma 2.5 (Uniqueness). Let $v \in L_{\text {loc }}^{1}(\bar{\Omega} \times[0, T])$ satisfy div $v=0$ in $\Omega \times(0, T), v \cdot N=0$ on $\partial \Omega \times(0, T)$ and

$$
\int_{0}^{T} \int_{\Omega} v \cdot\left(\partial_{t} \varphi+\Delta \varphi\right) d x d t=0
$$

for all $\varphi \in C_{c}^{2,1}(\bar{\Omega} \times[0, T])$ such that div $\varphi=0$ in $\Omega \times(0, T), \varphi=0$ on $\partial \Omega \times(0, T)$ and $\Omega \times\{t=T\}$. Assume that

$$
v \in L^{\infty}\left(0, T ; L^{p}\right) \quad \text { for } p \in(1, \infty)
$$

Then, $v \equiv 0$.

Proof. We first extend test functions so that solutions of (2.5) satisfy (2.8). We then apply a duality argument.

Step 1. We show that (2.8) holds for all $\varphi \in C^{2,1}(\bar{\Omega} \times[0, T])$ such that $\operatorname{div} \varphi=0$ in $\Omega \times(0, T), \varphi=0$ on $\partial \Omega \times(0, T) \cup \Omega \times\{t=T\}$,

$$
\partial_{t}^{s} \partial_{x}^{k} \varphi \in L^{\infty}\left(0, T ; L^{p^{\prime}}\right), \quad 2 s+|k| \leq 2,1 / p+1 / p^{\prime}=1
$$

We consider a cut-off function argument. Let $B_{0}\left(R_{0}\right)$ denote an open ball centered at the origin with radius $R_{0}>0$. We take $R_{0}>0$ so that $\Omega^{c} \subset B_{0}\left(R_{0}\right)$. Let $\theta \in C_{c}^{\infty}[0, \infty)$ be a function such that $\theta \equiv 1$ in $[0,1]$ and $\theta \equiv 0$ in $[2, \infty)$. We set $\theta_{R}(x)=\theta(|x| / R)$ for $R \geq R_{0}$. Since $\operatorname{div} \varphi=0$ in $\Omega$ and $\varphi=0$ on $\partial \Omega$, the average of $h_{R}=\varphi \cdot \nabla \theta_{R}$ in $D_{R}$ is zero. We set $w_{R}=B_{R}\left(h_{R}\right)$ by Proposition 2.4 (ii) and consider its zero extension to $\mathbb{R}^{n}$ (still denoted by $\left.w_{R}\right)$. Since $\varphi \in C^{2,1}(\bar{\Omega} \times[0, T])$, applying (2.7) implies that $w_{R} \in C\left([0, T] ; W^{3, q}\left(\mathbb{R}^{n}\right)\right)$ for $q \in(1, \infty)$. Thus $\nabla^{2} w_{R} \in C_{c}\left(\mathbb{R}^{2} \times[0, T]\right)$ by the Sobolev embedding. Since $\partial_{t} w_{R}=B_{R}\left(\partial_{t} h_{R}\right)$, we have $\partial_{t} w_{R} \in C_{c}\left(\mathbb{R}^{n} \times[0, T]\right)$. Thus $w_{R} \in C_{c}^{2,1}\left(\mathbb{R}^{n} \times[0, T]\right)$. We set

$$
\varphi_{R}=\varphi \theta_{R}-w_{R}
$$

so that $\varphi_{R} \in C_{c}^{2,1}(\bar{\Omega} \times[0, T])$ satisfies $\operatorname{div} \varphi_{R}=0$ in $\Omega$ and $\varphi_{R}=0$ on $\partial \Omega$ for $t \in[0, T]$. By substituting $\varphi_{R}$ into (2.8), we see that

$$
0=\int_{0}^{T} \int_{\Omega} v \cdot\left(\partial_{t} \varphi \theta_{R}+\Delta \varphi \theta_{R}+2 \nabla \varphi \cdot \nabla \theta_{R}+\varphi \Delta \theta_{R}\right) \mathrm{d} x \mathrm{~d} t-\int_{0}^{T} \int_{\Omega} v \cdot\left(\partial_{t} w_{R}+\Delta w_{R}\right) \mathrm{d} x \mathrm{~d} t .
$$

By (2.9) and (2.10), we have

$$
\lim _{R \rightarrow \infty} \int_{0}^{T} \int_{\Omega} v \cdot\left(\partial_{t} \varphi \theta_{R}+\Delta \varphi \theta_{R}+2 \nabla \varphi \cdot \nabla \theta_{R}+\varphi \Delta \theta_{R}\right) \mathrm{d} x \mathrm{~d} t=\int_{0}^{T} \int_{\Omega} v \cdot\left(\partial_{t} \varphi+\Delta \varphi\right) \mathrm{d} x \mathrm{~d} t
$$


Since $\partial_{t} w_{R}=B\left(\partial_{t} h_{R}\right)$, by the Poincaré inequality [28, 5.8.1 THEOREM] and (2.7) we estimate

$$
\left\|\partial_{t} w_{R}\right\|_{L^{p^{\prime}\left(D_{R}\right)}} \lesssim R\left\|\nabla \partial_{t} w_{R}\right\|_{L^{p^{\prime}\left(D_{R}\right)}}=R\left\|\nabla B_{R}\left(\partial_{t} h_{R}\right)\right\|_{L^{p^{\prime}\left(D_{R}\right)}} \lesssim R\left\|\partial_{t} h_{R}\right\|_{L^{p^{\prime}\left(D_{R}\right)}} \lesssim\left\|\partial_{t} \varphi\right\|_{L^{p^{\prime}\left(D_{R}\right)}} .
$$

In a similar way, we estimate $\left\|\nabla^{2} w_{R}\right\|_{L^{p^{\prime}\left(D_{R}\right)}} \lesssim\left\|\nabla h_{R}\right\|_{L^{p^{\prime}\left(D_{R}\right)}} \lesssim\|\varphi\|_{W^{1, p^{\prime}\left(D_{R}\right)}}$. It follows that

$$
\begin{aligned}
\left|\int_{0}^{T} \int_{\Omega} v \cdot\left(\partial_{t} w_{R}+\Delta w_{R}\right) \mathrm{d} x \mathrm{~d} t\right| & \lesssim\|v\|_{L^{\infty}\left(0, T ; L^{p}(\Omega)\right)}\left(\int_{0}^{T}\left(\left\|\partial_{t} \varphi\right\|_{L^{p^{\prime}}\left(D_{R}\right)}+\|\varphi\|_{W^{1, p^{\prime}}\left(D_{R}\right)}\right) \mathrm{d} t\right) \\
& \rightarrow 0, \quad \text { as } R \rightarrow \infty .
\end{aligned}
$$

Thus (2.8) holds for $\varphi$ satisfying (2.10).

Step 2. We apply a duality argument. Since $\operatorname{div} v=0$ in $\Omega, v \cdot N=0$ on $\partial \Omega$, we have

$$
\int_{\Omega} v(x, t) \cdot \nabla \Phi(x) \mathrm{d} x=0, \quad \text { a.e. } t \in(0, T)
$$

for all $\nabla \Phi \in G_{0}$. Since $G_{0}$ is dense in $G^{p^{\prime}}$ by Proposition 2.1 (i), this equality is extendable for all $\nabla \Phi \in G^{p^{\prime}}$. Hence, $v(\cdot, t) \in L_{\sigma}^{p}$ by $(2.1)$.

We take arbitrary $f_{0} \in C_{c, \sigma}^{\infty}(\Omega)$ and $\eta \in C_{c}^{\infty}(0, T)$. We take a solution $(\varphi, \pi)$ of $(2.5)$ for $\tilde{f}_{0}(x, t)=f_{0}(x) \eta(t)$ by Lemma 2.3. Since $\varphi$ satisfies (2.10), we have

$$
\int_{0}^{T} \eta(t)\left(\int_{\Omega} v(x, t) \cdot f_{0}(x) \mathrm{d} x\right) \mathrm{d} t=\int_{0}^{T} \int_{\Omega} v \cdot \tilde{f}_{0} \mathrm{~d} x \mathrm{~d} t=\int_{0}^{T} \int_{\Omega} v \cdot\left(\partial_{t} \varphi+\Delta \varphi-\nabla \pi\right) \mathrm{d} x \mathrm{~d} t=0 .
$$

Since $\eta$ is arbitrary, we have

$$
\int_{\Omega} v(x, t) \cdot f_{0}(x) \mathrm{d} x=0, \quad \text { a.e. } t \in(0, T)
$$

By taking closure of $C_{c, \sigma}^{\infty}$ in $L^{p^{\prime}}$, the above equality is extendable for all $f_{0} \in L_{\sigma}^{p^{\prime}}$. For an arbitrary $f \in C_{c}^{\infty}$, we set $f_{0}=\mathbb{P} f$ and $\nabla \Phi=(I-\mathbb{P}) f$ by Proposition 2.1 (i). Since $v(\cdot, t) \in L_{\sigma}^{p}$, it follows that

$$
\int_{\Omega} v(x, t) \cdot f(x) \mathrm{d} x=\int_{\Omega} v(x, t) \cdot\left(f_{0}(x)+\nabla \Phi(x)\right) \mathrm{d} x=0, \quad \text { a.e. } t \in(0, T)
$$

We proved $v \equiv 0$. The proof is now complete. 
2.3. Liouville theorems. We apply Lemma 2.5 to prove Theorem 1.1.

Proof of Theorem 1.1. We take an arbitrary $T>0$ and set $u(t)=v(t-T)$ for $t \in[0, T]$. By (1.7), $u$ satisfies

$$
\int_{0}^{T} \int_{\Omega} u \cdot\left(\partial_{t} \varphi+\Delta \varphi\right) \mathrm{d} x \mathrm{~d} t=-\int_{\Omega} u(x, 0) \cdot \varphi(x, 0) \mathrm{d} x
$$

for all $\varphi \in C_{c}^{2,1}(\bar{\Omega} \times[0, T])$, div $\varphi=0$ in $\Omega \times(0, T), \varphi=0$ on $\partial \Omega \times(0, T) \cup \Omega \times\{t=T\}$. We set $\tilde{u}(t)=S(t) u(0)$. Since $S(t)$ is a bounded analytic semigroup of angle $\pi / 2$ on $L_{\sigma}^{p}$, $\tilde{u}(t)$ is defined for $\operatorname{Re} t>0$ and bounded in $\Sigma_{\theta}$ for each $\theta \in(0, \pi / 2)$. Since $\tilde{u}(t)=S(t) u(0)$ also satisfies (2.11), applying Lemma 2.5 implies $u(t)=S(t) u(0)$. Thus $u(t)$ is uniquely continued for $\operatorname{Re} t>0$ and satisfies

$$
\sup \left\{\|u\|_{L^{p}}(t)|t \neq 0,| \arg t \mid \leq \theta\right\} \leq C\|u\|_{L^{p}}(0)
$$

Hence we have

$$
\sup \left\{\|v\|_{L^{p}}(t)|t \neq-T, \arg (t+T)| \leq \theta\right\} \leq C\|v\|_{L^{p}}(-T) .
$$

Since the right-hand side is uniformly bounded for $T>0$ by (1.8), by taking a supremum for $T>0$, the ancient solution is a bounded entire function on $L^{p}$. Thus, $\partial_{t} v \equiv 0$ by the classical Liouville theorem. Since $\partial_{t} v-A v=0$, we have $A v \equiv 0$. Since $\mathcal{N}\left(A_{p}\right)=\{0\}$ by Proposition 2.1 (iii), $v \equiv 0$ follows.

To prove (1.6) for complex time, we prepare a Liouville theorem for ancient solutions in the sector $\Lambda=\{t \in \mathbb{C} \backslash\{0\} \mid-\pi+\theta \leq \arg t \leq-\pi / 2\}$ for $\theta \in(0, \pi / 2)$.

Theorem 2.6. Let $v$ be an ancient solution to (1.1) in $\Omega \times \Lambda$ for $\theta \in(0, \pi / 2)$. Assume that

$$
v \in L^{\infty}\left(\Lambda ; L^{p}\right) \quad \text { for } p \in(1, \infty)
$$

Then, $v \equiv 0$.

Proof. We take an arbitrary $T>0$ and set $\tilde{T}=T+i T \tan \theta$. By translation, we set $u(t)=$ $v(t-\tilde{T})$ so that $u$ satisfies (1.1) in $\Omega \times(0, T)$. In fact, by (1.13) we have

$$
\int_{0}^{T} \int_{\Omega} u(x, t)\left(\partial_{t} \varphi+\Delta \varphi\right) \mathrm{d} x \mathrm{~d} t=-\int_{\Omega} u(x, 0) \varphi(x, 0) \mathrm{d} x
$$

for $\varphi \in C_{c}^{2,1}(\bar{\Omega} \times[0, T))$ satisfying $\operatorname{div} \varphi=0$ in $\Omega \times(0, T)$ and $\varphi=0$ on $\partial \Omega \times(0, T) \cup \Omega \times\{t=T\}$. 
Then by Lemma 2.5, we have $u(t)=S(t) u(0)$. Hence $u(t)$ is uniquely continued for $\operatorname{Re} t>0$ and bounded in $\Sigma_{\theta^{\prime}}$ for $\theta^{\prime} \in(\theta, \pi / 2)$, i.e.,

$$
\sup \left\{\|u\|_{L^{p}}(t)|t \neq 0,| \arg t \mid \leq \theta^{\prime}\right\} \leq C\|u\|_{L^{p}}(0)
$$

Since $v(t)=S(t+\tilde{T}) v(-\tilde{T}), v(t)$ is continued for $\operatorname{Re} t>-T$ and

$$
\sup \left\{\|v\|_{L^{p}}(t)|t \neq-\tilde{T},| \arg (t+\tilde{T}) \mid \leq \theta^{\prime}\right\} \leq C\|v\|_{L^{p}}(-\tilde{T})
$$

Since the right-hand side is uniformly bounded for $T>0$ by (2.12), $v(t)$ is a bounded entire function on $L^{p}$. Hence $v \equiv 0$ follows as we proved Theorem 1.1.

Remarks 2.7. (i) Liouville theorems for the Navier-Stokes equations are studied in [40] in connection with the regularity problem. It is proved in [40] that bounded ancient solutions $u \in L^{\infty}\left(\mathbb{R}^{n} \times(-\infty, 0)\right)$ must be constant for $n=2$ and for axisymmetric solutions without swirl. For the case with swirl, a Liouville theorem is proved under the decay condition

$$
|u(x, t)| \leq \frac{C}{\sqrt{x_{1}^{2}+x_{2}^{2}}}, \quad x \in \mathbb{R}^{3}, t \leq 0 .
$$

It is conjectured in [54] that any bounded ancient solutions is constant. See [52], [32], [9] for a half space. For stationary solutions, a Liouville theorem is known to hold under the condition $u \in L^{9 / 2}\left(\mathbb{R}^{3}\right)$ [30]. See, e.g., [21], [20], [53], [41], [55] and the references therein for further improvements.

(ii) For the Stokes flow, Liouville theorems are studied in [39] for domains. It is proved in [39] that bounded ancient solutions $v \in L^{\infty}(\Omega \times(-\infty, 0))$ must be trivial for $\Omega=\mathbb{R}^{n}, \mathbb{R}_{+}^{n}$ and bounded domains for $n \geq 2$. For exterior domains for $n \geq 3$, Liouville theorem does not hold for merely bounded ancient solutions due to existence of stationary solutions which are asymptotically constant as $|x| \rightarrow \infty$. See, e.g., [30]. It is shown in [39] that bounded ancient solutions $v(x, t)$ must satisfy

$$
v(x, t)-a(t)=O\left(|x|^{-n+2}\right) \quad \text { as }|x| \rightarrow \infty,
$$

for some constant $a(t)$. This characterization is based on the representation formula (1.9). We proved a Liouville theorem for exterior domains for $n \geq 2$ under the decay condition for the spatial variable $v \in L^{\infty}\left(-\infty, 0 ; L^{p}\right)$ for $p \in(1, \infty)$ based on the boundedness of the Stokes semigroup on $L_{\sigma}^{p}$ [15], [17]. 
3. Spatial decay estimates of the Stokes flow

We prepare a spatial decay estimate of the Stokes flow (Lemma 3.8) to prove (1.6) by a contradiction argument. To do this, we prove the representation formula (1.9). We regard $S(t) v_{0}$ as the Stokes flow in $\mathbb{R}^{n}$ with an external force and apply the Duhamel's principle.

3.1. The Stokes flow on $L^{\infty}$. We recall solutions of (1.1) for bounded data $v_{0} \in L_{\sigma}^{\infty}$. Solutions of (1.1) for $v_{0} \in L_{\sigma}^{\infty}$ are constructed by approximation by elements of $C_{c, \sigma}^{\infty}$.

Proposition 3.1. There exists $C>0$ such that for $v_{0} \in L_{\sigma}^{\infty}$ there exists $\left\{v_{0, m}\right\} \subset C_{c, \sigma}^{\infty}$ such that

$$
\begin{aligned}
\left\|v_{0, m}\right\|_{L^{\infty}} & \leq C\left\|v_{0}\right\|_{L^{\infty}}, \\
v_{0, m} & \rightarrow v_{0} \quad \text { a.e. } x \in \Omega \quad \text { as } m \rightarrow \infty .
\end{aligned}
$$

In particular, we have $L_{\sigma}^{\infty}=\left\{f \in L^{\infty} \mid\right.$ div $f=0$ in $\Omega, f \cdot N=0$ on $\left.\partial \Omega\right\}$. If $v_{0} \in B U C_{\sigma}$, $v_{0, m} \rightarrow v_{0}$ locally uniformly in $\bar{\Omega}$, where

$$
B U C_{\sigma}(\Omega)=\{f \in B U C(\bar{\Omega}) \mid \operatorname{div} f=0 \text { in } \Omega, f=0 \text { on } \partial \Omega\} \text {. }
$$

Proof. See [4, Lemma 5.1].

Proposition 3.2. (i) Let $T>0$. There exists $C>0$ such that

$$
\sup _{0<t \leq T}\left(t^{\frac{|k|}{2}+s}\left\|\partial_{t}^{s} \partial_{x}^{k} v\right\|_{L^{\infty}}(t)+t\|\nabla q\|_{\infty}(t)\right) \leq C\left\|v_{0}\right\|_{L^{\infty}}
$$

for $v=S(t) v_{0}$ and $\nabla q=(I-\mathbb{P}) \Delta v$ for $v_{0} \in C_{c, \sigma}^{\infty}$ and $2 s+|k| \leq 2$. The associated pressure $q$ satisfies

$$
\sup _{x \in \Omega} d(x)|\nabla q(x, t)| \leq C\|w\|_{L^{\infty}(\partial \Omega)}(t),
$$

for $w=-\left(\nabla v-\nabla^{T} v\right) N$ and $t>0$ with $d(x)=\inf \{|x-y| \mid y \in \partial \Omega\}$.

(ii) For $v_{0} \in L_{\sigma}^{\infty}$ and $v_{0, m} \in C_{c, \sigma}^{\infty}$ satisfying (3.1), $v_{m}=S(t) v_{0, m}$ subsequently converges to a limit $v$ locally uniformly in $\bar{\Omega} \times(0, \infty)$ together with $\partial_{t}^{s} \partial_{x}^{k} v_{m}$ and $\nabla q_{m}$ for $2 s+|k| \leq 2$.

Proof. The a priori estimates (3.2) and (3.3) are proved in [3] for admissible domains. See also [4, Lemmas 2.8, 2.12]. It is shown in [4, Theorem 3.1] that exterior domains of class $C^{3}$ are admissible. The assertion (ii) is based on (3.1), (3.2) and Hölder estimates for the Stokes equations [59], [60]. See the proof of Theorem 3.2 in [4]. 
Proposition 3.3. (i) For $v_{0} \in L_{\sigma}^{\infty}$, there exists a unique solution $(v, \nabla q) \in C^{2,1}(\bar{\Omega} \times(0, T]) \times$ $C(\bar{\Omega} \times(0, T])$ of (1.1) satisfying (3.2), (3.3), and $v(\cdot, t) \rightarrow v_{0}$ weakly-star on $L^{\infty}$ as $t \rightarrow 0$.

(ii) The solution operator $S(t): v_{0} \longmapsto v(\cdot, t)$ forms an analytic semigroup on $L_{\sigma}^{\infty}$ of angle $\pi / 2$ and satisfies

$$
\sup _{0<t \leq T} t^{\frac{|k|}{2}+s}\left\|\partial_{t}^{s} \partial_{x}^{k} S(t) v_{0}\right\|_{L^{\infty}} \leq C\left\|v_{0}\right\|_{L^{\infty}} .
$$

The semigroup $S(t)$ is weakly-star continuous on $L_{\sigma}^{\infty}$ at $t=0$ and strongly continuous on $B U C_{\sigma}$.

Proof. Proposition 3.2 (ii) implies existence of solutions to (1.1) for $v_{0} \in L_{\sigma}^{\infty}$. The uniqueness follows from a blow-up argument [4, Lemma 2.12]. Since (3.2) is inherited to $v,(3.4)$ follows. The estimate (3.4) implies analyticity of $S(t)$ on $L_{\sigma}^{\infty}$ [4, Theorem 3.2]. The angle $\pi / 2$ follows from a resolvent estimate on $L^{\infty}$ [5, Theorem 1.3].

Remark 3.4. (The Stokes operator on $L_{\sigma}^{\infty}$ ). We define the Stokes operator on $L_{\sigma}^{\infty}$ by

$$
\begin{aligned}
& A_{\infty} f=\lim _{t \rightarrow 0} \frac{S(t)-I}{t} f, \quad f \in D\left(A_{\infty}\right), \\
& D\left(A_{\infty}\right)=\left\{f \in L_{\sigma}^{\infty} \mid A_{\infty} f \in L_{\sigma}^{\infty}\right\} .
\end{aligned}
$$

The domain $D\left(A_{\infty}\right)$ is equipped with the graph-norm $\|f\|_{D\left(A_{\infty}\right)}=\|f\|_{L^{\infty}}+\left\|A_{\infty} f\right\|_{L^{\infty}}$. See also [27]. Although $A_{\infty}$ may not be represented by the projection $\mathbb{P}$, by the following Proposition 3.5 , we have $D\left(A_{\infty}\right) \subset W_{\mathrm{ul}}^{2, p}(\bar{\Omega})$ for $p>n$ with continuous injection. In the sequel, we write $A=A_{\infty}$ if there is no confusion.

Proposition 3.5. For $f \in L_{\sigma}^{\infty}$, there exists a unique solution $(v, \nabla q) \in W_{u l}^{2, p}(\bar{\Omega}) \times\left(L_{u l}^{p}(\bar{\Omega}) \cap L_{d}^{\infty}(\Omega)\right)$, $p>n$, of the Stokes equations

$$
\begin{aligned}
v-\Delta v+\nabla q=f, & \operatorname{div} v=0 & & \text { in } \Omega, \\
v & =0 & & \text { on } \partial \Omega,
\end{aligned}
$$

satisfying the estimate

$$
\|v\|_{W_{u l}^{2, p}(\bar{\Omega})}+\|\nabla q\|_{L_{u l}^{p}(\bar{\Omega})} \leq C\|f\|_{L^{\infty}(\Omega)}
$$

Here, $L_{u l}^{p}(\bar{\Omega})$ denotes the uniformly local $L^{p}$ space equipped with the norm

$$
\|g\|_{L_{u l}^{p}(\bar{\Omega})}=\sup \left\{\|g\|_{L^{p}\left(B_{x_{0}}(1) \cap \Omega\right)} \mid x_{0} \in \Omega\right\} .
$$

The space $W_{u l}^{2, p}(\bar{\Omega})$ is equipped with the norm $\|v\|_{W_{u l}^{2, p}(\bar{\Omega})}=\sum_{|k| \leq 2}\left\|\partial_{x}^{k} v\right\|_{L_{u l}^{p}(\bar{\Omega})}$ and $L_{d}^{\infty}(\Omega)$ denotes the space of functions $g \in L_{l o c}^{1}(\Omega)$ such that $d g \in L^{\infty}(\Omega)$. 
Proof. See [5, Theorem 1.1].

Remark 3.6. For domains with non-compact boundaries $\Omega \subset \mathbb{R}^{n}$, analyticity of $S(t)$ on $L_{\sigma}^{\infty}$ is also studied. See [1] for a perturbed half space $(n \geq 3)$, [6] for a sector-like domain $(n=2)$, and [7] for cylinders $(n \geq 2)$. For layers $(n \geq 3), S(t)$ may not be bounded on $L_{\sigma}^{\infty}[11],[64]$.

3.2. Spatial decay estimates. We estimate the stress tensor on $\partial \Omega$ by using the resolvent estimate (3.7). We then prove a spatial decay estimate of $S(t) v_{0}$ from the representation formula (1.9).

Proposition 3.7. Let $\Omega_{0}=\Omega \cap B_{0}\left(R_{0}\right)$ and $R_{0}>0$ such that $\Omega^{c} \subset B_{0}\left(R_{0}\right)$. There exists $C>0$ such that

$$
\sup _{0<s \leq t}\|T\|_{L^{\infty}(\partial \Omega)}(s) \leq C\left(\left\|v_{0}\right\|_{D(A)}+\sup _{0<s \leq t}\|v\|_{L^{\infty}(\Omega)}(s)\right), \quad t>0,
$$

for $T=\nabla v+\nabla^{T} v-q I, v=S(t) v_{0}, v_{0} \in D(A)$ and $q$ satisfying

$$
\int_{\Omega_{0}} q(x, t) d x=0
$$

Proof. Since the average of $q$ in $\Omega_{0}$ is zero, by the Poincaré inequality [28, 5.8.1 THEOREM 1], we estimate

$$
\|q\|_{L^{p}\left(\Omega_{0}\right)} \leq C\|\nabla q\|_{L^{p}\left(\Omega_{0}\right)} \leq C^{\prime}\|\nabla q\|_{L_{\mathrm{ul}}^{p}(\bar{\Omega})} .
$$

By the Sobolev inequality for $p>n$, we estimate

$$
\|q\|_{L^{\infty}\left(\Omega_{0}\right)} \leq C\|q\|_{W^{1, p}\left(\Omega_{0}\right)} \leq\|\nabla q\|_{L_{\mathrm{ul}}^{p}(\bar{\Omega})} .
$$

Since $v=S(s) v_{0}$ satisfies (3.6) for $f=v+A v$ and $D(A) \subset W_{\mathrm{ul}}^{2, p}(\bar{\Omega}) \subset W^{1, \infty}(\Omega)$ by Remark 3.3, we have

$$
\|T\|_{L^{\infty}(\partial \Omega)} \leq C\left(\|v\|_{W_{\mathrm{ul}}^{2, p}(\bar{\Omega})}+\|\nabla q\|_{L_{\mathrm{ul}}^{p}(\bar{\Omega})}\right) \leq C^{\prime}\|v\|_{D(A)}
$$

We estimate the right-hand side for $s \leq t$. We may assume that $t>1$. For $s \in(0,1)$, we apply (3.4) to estimate

$$
\|v\|_{D(A)}(s)=\left\|S(s) v_{0}\right\|_{L^{\infty}}+\left\|A S(s) v_{0}\right\|_{L^{\infty}} \leq C\left(\left\|v_{0}\right\|_{L^{\infty}}+\left\|A v_{0}\right\|_{L^{\infty}}\right)=C\left\|v_{0}\right\|_{D(A)} .
$$


For $s \in[1, t]$, we have

$$
\begin{aligned}
\|v\|_{D(A)}(s) & =\left\|S(s) v_{0}\right\|_{L^{\infty}}+\left\|A S(1) S(s-1) v_{0}\right\|_{L^{\infty}} \\
& \leq\left\|S(s) v_{0}\right\|_{L^{\infty}}+C\left\|S(s-1) v_{0}\right\|_{L^{\infty}} \leq C^{\prime} \sup _{0<s \leq t}\|v\|_{L^{\infty}}(s) .
\end{aligned}
$$

Thus (3.8) holds.

Lemma 3.8 (Spatial decay estimate). (i) Let $v=S(t) v_{0}$ and $v_{0} \in D(A)$. Then,

$$
v(x, t)=\int_{\Omega} \Gamma(x-y, t) v_{0}(y) d y+\int_{0}^{t} \int_{\partial \Omega} V(x-y, t-s) T(y, s) N(y) d H(y) d s,
$$

for $x \in \Omega$ and $t>0$.

(ii) Let $n \geq 3$. There exists $C>0$ such that

$$
|v(x, t)| \leq\left\|v_{0}\right\|_{L^{\infty}}+\frac{C}{d(x)^{n-2}} \sup _{0<s \leq t}\|T\|_{L^{\infty}(\partial \Omega)}(s), \quad x \in \Omega, t>0 .
$$

If $q$ satisfies (3.9), then

$$
|v(x, t)| \leq\left\|v_{0}\right\|_{L^{\infty}}+\frac{C}{d(x)^{n-2}}\left(\left\|v_{0}\right\|_{D(A)}+\sup _{s>0}\|v\|_{L^{\infty}}(s)\right), \quad x \in \Omega, t>0 .
$$

Proof. By (1.11), we estimate

$$
\begin{aligned}
\left|\int_{0}^{t} \int_{\partial \Omega} V(x-y, t-s) T(y, s) N(y) \mathrm{d} H(y) \mathrm{d} s\right| & \lesssim \sup _{0<s \leq t}\|T\|_{L^{\infty}(\partial \Omega)}(s) \int_{0}^{t} \int_{\partial \Omega} \frac{\mathrm{d} H(y) \mathrm{d} s}{\left(|x-y|+s^{1 / 2}\right)^{n}} \\
& \lesssim \frac{1}{d(x)^{n-2}} \sup _{0<s \leq t}\|T\|_{L^{\infty}(\partial \Omega)}(s) \int_{0}^{\infty} \frac{\mathrm{d} s}{\left(1+s^{1 / 2}\right)^{n}} .
\end{aligned}
$$

The right-hand side is finite for $n \geq 3$. Thus (3.11) follows from (3.10). The estimate (3.12) follows from (3.11) and (3.8).

We prove (3.10). Let $(\bar{v}, \bar{q})$ be the zero extension of $(v, q)$ to $\mathbb{R}^{n}$. Then, $(\bar{v}, \bar{q})$ satisfies

$$
\begin{aligned}
& \partial_{t} \bar{v}-\Delta \bar{v}+\nabla \bar{q}=\mu, \quad \operatorname{div} \bar{v}=0 \quad \text { in } \mathbb{R}^{n} \times(0, \infty), \\
& \bar{v}=\bar{v}_{0} \quad \text { on } \mathbb{R}^{n} \times\{t=0\},
\end{aligned}
$$

for a measure $\mu(\cdot, t) \in M\left(\mathbb{R}^{n}\right)$ such that

$$
(\mu, \varphi)=\int_{\partial \Omega} T(y, t) N(y) \varphi(y) \mathrm{d} H(y), \quad \varphi \in C_{0}\left(\mathbb{R}^{n}\right) .
$$


See [39]. Here, $C_{0}\left(\mathbb{R}^{n}\right)$ is the $L^{\infty}$-closure of $C_{c}^{\infty}\left(\mathbb{R}^{n}\right)$ and $M\left(\mathbb{R}^{2}\right)$ is the space of finite regular Borel measures on $\mathbb{R}^{n}$, equipped with the total variation. The bracket $(\cdot, \cdot)$ denotes the pairing for $C_{0}$ and the adjoint space $M$. Indeed, by integration by parts, $(\bar{v}, \bar{q})$ satisfies (3.13) in the sense that

$$
\frac{\mathrm{d}}{\mathrm{d} t} \int_{\mathbb{R}^{n}} \bar{v} \cdot \varphi \mathrm{d} y-\int_{\mathbb{R}^{n}} \bar{v} \cdot \Delta \varphi \mathrm{d} y-\int_{\mathbb{R}^{n}} \bar{q} \operatorname{div} \varphi \mathrm{d} y=\int_{\partial \Omega} T N \cdot \varphi \mathrm{d} H(y), \quad \varphi \in C_{c}^{\infty}\left(\mathbb{R}^{n}\right) .
$$

Since $T \in L^{\infty}\left(\partial \Omega \times\left(0, T_{0}\right)\right)$ for $T_{0}>0$ by (3.8), we have $\mu \in L^{\infty}\left(0, T_{0} ; M\right)$. We set $v_{\varepsilon}=\eta_{\varepsilon} * \bar{v}$, $q_{\varepsilon}=\eta_{\varepsilon} * \bar{q}$ and $\mu_{\varepsilon}=\eta_{\varepsilon} * \mu$ with the mollifier $\eta_{\varepsilon} \in C_{c}^{\infty}\left(\mathbb{R}^{n}\right)$. Then, by substituting $\eta_{\varepsilon}(x-y)$ into the above, we have

$$
\begin{aligned}
& \partial_{t} v_{\varepsilon}-\Delta v_{\varepsilon}+\nabla q_{\varepsilon}=\mu_{\varepsilon}, \quad \operatorname{div} v_{\varepsilon}=0 \quad \text { in } \mathbb{R}^{n} \times(0, \infty), \\
& v_{\varepsilon}=v_{0, \varepsilon} \quad \text { on } \mathbb{R}^{n} \times\{t=0\} .
\end{aligned}
$$

Since $\mu_{\varepsilon} \in L^{\infty}\left(0, T ; L^{p}\left(\mathbb{R}^{n}\right)\right)$ for $p \in[1, \infty]$ and $V(x, t)$ is the kernel of $e^{t \Delta} \mathbb{P}$, applying the Duhamel's principle implies

$$
\begin{aligned}
v_{\varepsilon}(x, t) & =e^{t \Delta} v_{0, \varepsilon}+\int_{0}^{t} e^{(t-s) \Delta} \mathbb{P} \mu_{\varepsilon}(s) \mathrm{d} s \\
& =\eta_{\varepsilon} *\left(\int_{\Omega} \Gamma(\cdot-y, t) v_{0}(y) \mathrm{d} y+\int_{0}^{t} \int_{\partial \Omega} V(\cdot-y, t-s) T(y, s) N(y) \mathrm{d} y \mathrm{~d} s\right) .
\end{aligned}
$$

The right-hand side converges locally uniformly in $\Omega$ for each $t>0$. Since $v_{\varepsilon} \rightarrow v$ locally uniformly in $\Omega$, sending $\varepsilon \rightarrow 0$ yields (3.10).

3.3. The case for complex time. We extend (3.12) for complex time $0 \leq \arg t \leq \theta$.

Lemma 3.9. Let $\gamma=\{t \in \mathbb{C} \backslash\{0\} \mid \arg t=\theta\}$ for $\theta \in(0, \pi / 2)$. We set $\gamma_{t}=\{s \in \gamma|| s|\leq| t \mid\}$ for $t \in \gamma$.

(i) There exists $C>0$ such that

$$
\sup _{s \in \gamma_{t}}\|T\|_{L^{\infty}(\partial \Omega)}(s) \leq C\left(\left\|v_{0}\right\|_{D(A)}+\sup _{s \in \gamma_{t}}\|v\|_{L^{\infty}}(s)\right), \quad t \in \gamma,
$$

for $v=S(t) v_{0}, v_{0} \in D(A)$ and $q$ satisfying (3.9).

(ii) The formula

$$
v(x, t)=\int_{\Omega} \Gamma(x-y, t) v_{0}(y) d y+\int_{\gamma_{t}} \int_{\partial \Omega} V(x-y, t-s) T(y, s) N(y) d H(y) d s,
$$

holds for $x \in \Omega$ and $t \in \gamma$. The second term is the line integral on $\gamma_{t}$.

(iii) Let $n \geq 3$. There exists $C>0$ such that 


$$
|v(x, t)| \leq C\left(\left\|v_{0}\right\|_{L^{\infty}}+\frac{1}{d(x)^{n-2}} \sup _{s \in \gamma_{t}}\|T\|_{L^{\infty}(\partial \Omega)}(s)\right), \quad x \in \Omega, t \in \gamma .
$$

If (3.9) holds, then

$$
|v(x, t)| \leq C\left\{\left\|v_{0}\right\|_{L^{\infty}}+\frac{1}{d(x)^{n-2}}\left(\left\|v_{0}\right\|_{D(A)}+\sup \left\{\|v\|_{L^{\infty}}(s) \mid 0 \leq \arg s \leq \theta\right\}\right)\right\},
$$

for $x \in \Omega$ and $0 \leq \arg t \leq \theta$.

Proof. The estimate (3.15) follows in the same way as (3.8) by using the semigroup property

$$
S(t+s)=S(t) S(s), \quad t, s \in \Sigma_{\theta} .
$$

Since $\left(v_{\varepsilon}, q_{\varepsilon}\right)$ solves (3.14) in $\mathbb{R}^{n} \times \gamma$, applying the Duhamel's principle on $\gamma_{t}$ yields (3.16). Since the Oseen tensor satisfies

$$
|V(x, t)| \leq \frac{C_{\theta}}{\left(|x|+|t|^{1 / 2}\right)^{n}}, \quad x \in \mathbb{R}^{n}, t \in \Sigma_{\theta},
$$

(3.17) follows from (3.16). We set $\gamma^{\prime}=\left\{\arg t=\theta^{\prime}\right\}$ for $\theta^{\prime} \in[0, \theta]$. By (3.17) and (3.15), we have

$$
\begin{aligned}
|v(x, t)| & \leq C\left\{\left\|v_{0}\right\|_{L^{\infty}}+\frac{1}{d(x)^{n-2}}\left(\left\|v_{0}\right\|_{D(A)}+\sup _{s \in \gamma^{\prime}}\|v\|_{L^{\infty}}(s)\right)\right\} \\
& \leq C\left\{\left\|v_{0}\right\|_{L^{\infty}}+\frac{1}{d(x)^{n-2}}\left(\left\|v_{0}\right\|_{D(A)}+\sup \left\{\|v\|_{L^{\infty}}(s) \mid 0 \leq \arg s \leq \theta\right\}\right)\right\}
\end{aligned}
$$

for $x \in \Omega, t \in \gamma^{\prime}$. By taking a supremum for $\theta^{\prime} \in[0, \theta]$, we obtain (3.18).

Lemma 3.10. For $n=2$, we have

$$
\begin{array}{r}
|v(x, t)| \leq\left\|v_{0}\right\|_{L^{\infty}}+\frac{C}{|x|}\left(\left\|v_{0}\right\|_{D(A)}+\sup _{s>0}\|v\|_{L^{\infty}}(s)\right), \quad|x| \geq 2 R_{0}, t>0, \\
|v(x, t)| \leq C\left\{\left\|v_{0}\right\|_{L^{\infty}}+\frac{1}{|x|}\left(\left\|v_{0}\right\|_{D(A)}+\sup \left\{\|v\|_{L^{\infty}}(s) \mid 0 \leq \arg s \leq \theta\right\}\right)\right\}, \\
|x| \geq 2 R_{0}, 0 \leq \arg t \leq \theta,
\end{array}
$$

for $v=S(t) v_{0}, v_{0} \in D(A)$ and $q$ satisfying (3.9) with zero net force 


$$
F(s)=\int_{\partial \Omega} T(y, s) N(y) d H(y) \equiv 0 .
$$

Proof. Since the Oseen tensor satisfies

$$
|\nabla V(x, t)| \leq \frac{C_{\theta}}{\left(|x|+|t|^{1 / 2}\right)^{3}} \quad x \in \mathbb{R}^{n}, t \in \Sigma_{\theta},
$$

by a simple calculation, we have

$$
|V(x-y, t)-V(x, t)| \leq \frac{C}{\left(|x|+|t|^{1 / 2}\right)^{3}}, \quad|x| \geq 2 R_{0}, y \in \partial \Omega .
$$

Hence

$$
\begin{aligned}
& \left|\int_{\gamma_{t}} \int_{\partial \Omega} V(x-y, t-s) T(y, s) N(y) \mathrm{d} H(y) \mathrm{d} s\right| \\
& =\left|\int_{\gamma_{t}} \int_{\partial \Omega}(V(x-y, t-s)-V(x, t-s)) T(y, s) N(y) \mathrm{d} H(y) \mathrm{d} s\right| \\
& \lesssim \sup _{s \in \gamma_{t}}\|T\|_{L^{\infty}(\partial \Omega)}(s) \int_{0}^{|t|} \frac{\mathrm{d} r}{\left(|x|+(|t|-r)^{1 / 2}\right)^{3}} \lesssim \frac{1}{|x|} \sup _{s \in \gamma_{t}}\|T\|_{L^{\infty}(\partial \Omega)}(s) \int_{0}^{\infty} \frac{\mathrm{d} r}{\left(1+r^{1 / 2}\right)^{3}} .
\end{aligned}
$$

Since (3.8) and (3.10) hold also for $n=2$, (3.19) follows in the same way as (3.12). Since (3.15) and (3.16) hold for $n=2$, (3.20) follows.

\section{The LARGe TIME ESTIMATE FOR POSITIVE TIME}

We prove the estimate (1.6) for $t>0$. We first prove a large time estimate of $S(t) v_{0}$ for $v_{0} \in D(A) \cap L^{2}$. Since $D(A) \cap L^{2} \subset L_{\sigma}^{\infty} \cap L^{2} \subset L_{\sigma}^{p}$ for $p \in[2, \infty]$, by (2.3) and the Sobolev embedding we have

$$
\lim _{t \rightarrow \infty}\left\|S(t) v_{0}\right\|_{L^{\infty}}=0
$$

In particular, $\left\|S(t) v_{0}\right\|_{L^{\infty}}$ is bounded for all $t>0$.

Lemma 4.1. There exists $C>0$ such that

$$
\sup _{t>0}\left\|S(t) v_{0}\right\|_{L^{\infty}}(t) \leq C\left\|v_{0}\right\|_{D(A)}, \quad v_{0} \in D(A) \cap L^{2} .
$$


Proof. We argue by a contradiction. Suppose on the contrary that (4.1) were false. Then, for any $m \geq 1$ there exists $\tilde{v}_{0, m} \in D(A) \cap L^{2}$ such that

$$
M_{m}:=\sup _{t>0}\left\|\tilde{v}_{m}\right\|_{L^{\infty}}(t)>m\left\|\tilde{v}_{0, m}\right\|_{D(A)}
$$

for $\tilde{v}_{m}=S(t) \tilde{v}_{0, m}$. We set $v_{m}=\tilde{v}_{m} / M_{m}$ so that

$$
\sup _{t>0}\left\|v_{m}\right\|_{L^{\infty}}(t)=1, \quad\left\|v_{0, m}\right\|_{D(A)}<\frac{1}{m} .
$$

We normalize the associated pressure $q_{m}$ so that

$$
\int_{\Omega_{0}} q_{m}(x, t) \mathrm{d} x=0
$$

for $\Omega_{0}=B_{0}\left(R_{0}\right) \cap \Omega$ and $R_{0}>0$ such that $\Omega^{c} \subset B_{0}\left(R_{0}\right)$. Then by (3.12), we have

$$
\left|v_{m}(x, t)\right| \leq \frac{1}{m}+\frac{C}{d(x)^{n-2}}\left(\frac{1}{m}+1\right), \quad x \in \Omega, t>0,
$$

with some constant $C$, independent of $m$. We take $t_{m} \in(0, \infty)$ such that $\left\|v_{m}\right\|_{L^{\infty}}\left(t_{m}\right) \geq 1 / 2$. By (3.4), we may assume that $t_{m} \rightarrow \infty$. We take $x_{m} \in \Omega$ such that

$$
\left|v_{m}\left(x_{m}, t_{m}\right)\right| \geq \frac{1}{4}
$$

Case 1. $\varlimsup_{m \rightarrow \infty} d\left(x_{m}\right)=\infty$. We may assume that $\lim _{m \rightarrow \infty} d\left(x_{m}\right)=\infty$ by choosing a subsequence. By (4.2), we see that

$$
\frac{1}{4} \leq\left|v_{m}\left(x_{m}, t_{m}\right)\right| \leq \frac{1}{m}+\frac{C}{d\left(x_{m}\right)^{n-2}}\left(\frac{1}{m}+1\right) \rightarrow 0, \quad \text { as } m \rightarrow \infty .
$$

Thus Case 1 does not occur.

Case 2. $\varlimsup_{m \rightarrow \infty} d\left(x_{m}\right)<\infty$. We may assume that $x_{m} \rightarrow x_{\infty} \in \bar{\Omega}$ by choosing a subsequence. We set

$$
u_{m}(x, t)=v_{m}\left(x, t+t_{m}\right), \quad p_{m}(x, t)=q_{m}\left(x, t+t_{m}\right) .
$$

Then, $\left(u_{m}, p_{m}\right)$ is a solution of (1.1) in $\Omega \times\left(-t_{m}, 0\right]$. By (4.2), we have

$$
\left|u_{m}(x, t)\right| \leq \frac{1}{m}+\frac{C}{d(x)^{n-2}}\left(\frac{1}{m}+1\right), \quad x \in \Omega, t>-t_{m} .
$$

Since $u_{m}$ is bounded in $\Omega \times\left(-t_{m}, 0\right], \partial_{t}^{s} \partial_{x}^{k} u_{m}$ are bounded in $\bar{\Omega} \times(-T, 0]$ for $2 s+|k| \leq 2$ 
and $T>0$ by (3.4). Thus, there exists a subsequence (still denoted by $u_{m}$ ) such that $u_{m}$ converges to a limit $u$ locally uniformly in $\bar{\Omega} \times(-\infty, 0]$. By sending $m \rightarrow \infty$, we have

$$
|u(x, t)| \leq \frac{C}{d(x)^{n-2}} \quad x \in \Omega, t \in \mathbb{R} .
$$

It is not difficult to see that the limit $u$ is an ancient solution to (1.1). We take $\varphi \in C_{c}^{2,1}(\bar{\Omega} \times$ $(-\infty, 0])$ satisfying $\operatorname{div} \varphi=0$ in $\Omega \times(-\infty, 0)$ and $\varphi=0$ on $\partial \Omega \times(-\infty, 0) \cup \Omega \times\{t=0\}$. Since $u_{m}$ satisfies (1.1) in $\Omega \times\left(-t_{m}, 0\right]$ and $\varphi$ is supported in $\bar{\Omega} \times\left(-t_{m}, 0\right]$ for sufficiently large $m$, by multiplying $\varphi$ by (1.1) and integration by parts, we have

$$
\int_{-t_{m}}^{0} \int_{\Omega} u_{m} \cdot\left(\partial_{t} \varphi+\Delta \varphi\right) \mathrm{d} x \mathrm{~d} t=0
$$

Sending $m \rightarrow \infty$ implies (1.7). The limit $u$ also satisfies div $u=0$ in $\Omega \times(-\infty, 0)$ and $u \cdot N=0$ on $\partial \Omega \times(-\infty, 0)$. Since $u \in L^{\infty}\left(-\infty, 0 ; L^{p}\right)$ for $p \in(n /(n-2), \infty)$ by (4.3), applying the Liouville theorem (Theorem 1.1) implies that $u \equiv 0$. This contradicts $\left|u\left(x_{\infty}, 0\right)\right| \geq 1 / 4$. Thus Case 2 does not occur.

We reached a contradiction. The proof is now complete.

\section{Lemma 4.2. There exists $C>0$ such that}

$$
\sup _{t>0}\left\|S(t) v_{0}\right\|_{L^{\infty}} \leq C\left\|v_{0}\right\|_{L^{\infty}}, \quad v_{0} \in L_{\sigma}^{\infty} .
$$

Proof. It suffices to show (4.4) for $v_{0} \in C_{c, \sigma}^{\infty}$ by Proposition 3.2 (ii). Since $S(t) v_{0}$ is bounded on $L_{\sigma}^{\infty}$ for $t \in(0,1]$ by (3.3), we consider the case $t \geq 1$. Since $S(1) v_{0} \in D(A) \cap L^{2}$ and

$$
\left\|S(1) v_{0}\right\|_{D(A)}=\left\|S(1) v_{0}\right\|_{\infty}+\left\|A S(1) v_{0}\right\|_{\infty} \leq C\left\|v_{0}\right\|_{L^{\infty}},
$$

we apply (4.1) to estimate

$$
\left\|S(t) v_{0}\right\|_{L^{\infty}}=\left\|S(t-1) S(1) v_{0}\right\|_{L^{\infty}} \leq C\left\|S(1) v_{0}\right\|_{D(A)} \leq C^{\prime}\left\|v_{0}\right\|_{L^{\infty}} .
$$

We proved (4.4).

\section{EXTENSIONS TO COMPLEX TIME}

We prove Theorem 1.2. We begin with a maximum principle in a sector. 
Proposition 5.1. Let $\theta \in(0, \pi / 2)$ and $\gamma=\{t \in \mathbb{C} \backslash\{0\} \mid \arg t=\theta\}$. For $v_{0} \in D(A) \cap L^{2}$,

$$
\begin{aligned}
& \lim _{|t| \rightarrow \infty} \sup \left\{\left\|S(t) v_{0}\right\|_{L^{\infty}} \mid \arg t=\theta\right\}=0, \\
& \sup \left\{\left\|S(t) v_{0}\right\|_{L^{\infty}} \mid 0 \leq \arg t \leq \theta\right\} \leq \max \left\{\sup _{t>0}\left\|S(t) v_{0}\right\|_{L^{\infty}}, \sup _{t \in \gamma}\left\|S(t) v_{0}\right\|_{L^{\infty}}\right\} .
\end{aligned}
$$

Proof. We shall show that

$$
\lim _{T \rightarrow \infty} \sup \left\{\left\|S(t) v_{0}\right\|_{L^{p}} \mid \operatorname{Re} t=T, 0 \leq \arg t \leq \theta\right\}=0, \quad p \in[2, \infty] .
$$

The property (5.1) follows from (5.3). Since $v_{0} \in D(A), S(t) v_{0}$ is strongly continuous on $L^{\infty}$ at $t=0$. Since $S(t) v_{0}$ is holomorphic in $\Sigma_{\theta}$ and continuous in $\overline{\Sigma_{\theta}}$, (5.2) follows from (5.3) and the maximum principle.

We take $\theta^{\prime} \in(\theta, \pi / 2)$. For $\operatorname{Re} t=T, 0 \leq \arg t \leq \theta$, there exists $t_{1}>0$ and $t_{2}$ such that $t=t_{1}+t_{2}$ and

$$
t_{1} \geq T\left(1-\frac{\tan \theta}{\tan \theta^{\prime}}\right), \quad \arg t_{2}=\theta^{\prime}
$$

Since $S\left(t_{2}\right) v_{0}$ is bounded on $L^{p}$ for $t_{2} \in \overline{\Sigma_{\theta^{\prime}}}$, by $S(t)=S\left(t_{2}\right) S\left(t_{1}\right)$ and (2.3) we have

$$
\begin{aligned}
\sup \left\{\left\|S(t) v_{0}\right\|_{L^{p}} \mid \operatorname{Re} t=T, 0 \leq \arg t \leq \theta\right\} & \leq C \sup \left\{\left\|S\left(t_{1}\right) v_{0}\right\|_{L^{p}} \mid T\left(1-\frac{\tan \theta}{\tan \theta^{\prime}}\right) \leq t_{1} \leq T\right\} \\
& \rightarrow 0 \text { as } T \rightarrow \infty .
\end{aligned}
$$

Thus (5.3) holds for $p \in[2, \infty)$. Since $D\left(A_{p}\right) \subset W^{2, p}$ by Proposition 2.1 (ii), by the Sobolev embedding, we estimate

$$
\left\|S(t) v_{0}\right\|_{L^{\infty}} \leq C\left\|S(t) v_{0}\right\|_{W^{1, p}} \leq C^{\prime}\left(\left\|S(t) v_{0}\right\|_{L^{p}}+\left\|A_{p} S(t) v_{0}\right\|_{L^{p}}\right) .
$$

Since $\left\|A_{p} S(t) v_{0}\right\|_{L^{p}}=\left\|A_{p} S(1) S(t-1) v_{0}\right\|_{L^{p}} \leq C\left\|S(t-1) v_{0}\right\|_{L^{p}},(5.3)$ holds for $p=\infty$.

We prove (4.1) on the half line $\gamma$.

Proposition 5.2. There exists $C>0$ such that

$$
\sup _{t \in \gamma}\left\|S(t) v_{0}\right\|_{L^{\infty}} \leq C\left\|v_{0}\right\|_{D(A)}, \quad v_{0} \in D(A) \cap L^{2} .
$$


Proof. Suppose that (5.4) were false. Then, for $m \geq 1$ there exisits $v_{0, m} \in D(A) \cap L^{2}$ such that

$$
\sup _{t \in \gamma}\left\|v_{m}\right\|_{L^{\infty}}(t)=1, \quad\left\|v_{0, m}\right\|_{D(A)}<\frac{1}{m},
$$

for $v_{m}=S(t) v_{0, m}$. Since $\sup _{t>0}\left\|v_{m}\right\|_{L^{\infty}} \leq 1$ for sufficiently large $m$ by (4.4), we apply (5.2) to estimate

$$
\sup \left\{\left\|v_{m}\right\|_{L^{\infty}}(t) \mid 0 \leq \arg t \leq \theta\right\} \leq 1 .
$$

We normalize the pressure $q_{m}$ so that (3.9) is satisfied. By (3.18), we have

$$
\left|v_{m}(x, t)\right| \leq C\left\{\frac{1}{m}+\frac{1}{d(x)^{n-2}}\left(\frac{1}{m}+1\right)\right\}, \quad x \in \Omega, 0 \leq \arg t \leq \theta
$$

with some constant $C$, independent of $m$. We take $t_{m} \in \gamma$ such that $\left\|v_{m}\right\|_{L^{\infty}}\left(t_{m}\right) \geq 1 / 2$. We may assume that $\left|t_{m}\right| \rightarrow \infty$ by Proposition 3.3 (ii). We take $x_{m} \in \Omega$ such that $\left|v_{m}\left(x_{m}, t_{m}\right)\right| \geq$ $1 / 4$.

Case 1. $\overline{\lim }_{m \rightarrow \infty} d\left(x_{m}\right)=\infty$. We may assume that $\lim _{m \rightarrow \infty} d\left(x_{m}\right)=\infty$. By (5.6), we have

$$
\frac{1}{4} \leq\left|v\left(x_{m}, t_{m}\right)\right| \leq C\left\{\frac{1}{m}+\frac{1}{d\left(x_{m}\right)^{n-2}}\left(\frac{1}{m}+1\right)\right\} \rightarrow 0, \quad \text { as } m \rightarrow \infty .
$$

Thus Case 1 does not occur.

Case 2. $\varlimsup_{m \rightarrow \infty} d\left(x_{m}\right)<\infty$. We may assume that $x_{m} \rightarrow x_{\infty} \in \bar{\Omega}$ by choosing a subsequence. We set

$$
u_{m}(x, t)=v_{m}\left(x, t+t_{m}\right), \quad p_{m}(x, t)=q_{m}\left(x, t+t_{m}\right) .
$$

Then $\left(u_{m}, p_{m}\right)$ satisfies (1.1) in $\Omega \times \Lambda_{m}$ for

$$
\Lambda_{m}=\left\{t \in \mathbb{C} \backslash\{0\}\left|t \neq-t_{m},-\pi+\theta \leq \arg t \leq-\frac{\pi}{2}, \operatorname{Im} t \geq-\right| t_{m} \mid \sin \theta\right\} .
$$

Since $\left|t_{m}\right| \rightarrow \infty$, the domain $\Lambda_{m}$ approaches to the sector

$$
\Lambda=\left\{t \in \mathbb{C} \backslash\{0\} \mid-\pi+\theta \leq \arg t \leq-\frac{\pi}{2}\right\} .
$$

By (5.6), we have 


$$
\left|u_{m}(x, t)\right| \leq C\left\{\frac{1}{m}+\frac{1}{d(x)^{n-2}}\left(\frac{1}{m}+1\right)\right\}, \quad x \in \Omega, t \in \Lambda_{m} .
$$

Since $\partial_{t}^{s} \partial_{x}^{k} u_{m}$ for $2 s+|k| \leq 2$ are uniformly bounded for each bounded domain in $\bar{\Omega} \times \bar{\Lambda}$ by Proposition 3.3 (ii), there exists a subsequence (still denoted by $u_{m}$ ) such that $u_{m}$ converges to a limit $u$ locally uniformly in $\bar{\Omega} \times \bar{\Lambda}$. For each $T>0$, we set

$$
I_{T}=\{t \in \Lambda \mid-T \leq \operatorname{Re} t \leq 0, \operatorname{Im} t=-T \tan \theta\} .
$$

Since $I_{T} \subset \Lambda_{m}$ for sufficiently large $m,\left(u_{m}, p_{m}\right)$ satisfies (1.1) in $\Omega \times I_{T}$. We take an arbitrary $\varphi \in C_{c}^{2,1}(\bar{\Omega} \times[-T, 0])$ satisfying $\operatorname{div} \varphi=0$ in $\Omega \times[-T, 0]$ and $\varphi=0$ on $\partial \Omega \times(-T, 0) \cup \Omega \times\{t=$ $0\}$. By multiplying $\varphi$ by (1.1) in $\Omega \times I_{T}$ and integration by parts, it follows that

$$
\int_{-T}^{0} \int_{\Omega} u_{m}(x, \alpha+i \beta)\left(\partial_{\alpha} \varphi+\Delta \varphi\right) \mathrm{d} x \mathrm{~d} \alpha=-\int_{\Omega} u_{m}(x,-T+i \beta) \varphi(x,-T+i \beta) \mathrm{d} x, \quad \beta=-T \tan \theta .
$$

Sending $m \rightarrow \infty$ implies that

$$
\int_{-T}^{0} \int_{\Omega} u(x, \alpha+i \beta)\left(\partial_{\alpha} \varphi+\Delta \varphi\right) \mathrm{d} x \mathrm{~d} \alpha=-\int_{\Omega} u(x,-T+i \beta) \varphi(x,-T+i \beta) \mathrm{d} x .
$$

Since div $u=0$ in $\Omega \times \Lambda$ and $u \cdot N=0$ on $\partial \Omega \times \Lambda$, the limit $u$ is an ancient solution in $\Omega \times \Lambda$. Since $u \in L^{\infty}\left(\Lambda ; L^{p}\right)$ for $p \in(n /(n-2), \infty)$ by (5.7), applying the Liouville theorem (Theorem 2.6) implies $u \equiv 0$. This contradicts $\left|u\left(x_{\infty}, 0\right)\right| \geq 1 / 4$. Thus Case 2 does not occur. We reached a contradiction. The proof is now complete.

Lemma 5.3. There exists $C>0$ such that

$$
\sup _{t \in \Sigma_{\theta}}\left\|S(t) v_{0}\right\|_{L^{\infty}} \leq C\left\|v_{0}\right\|_{L^{\infty}}, \quad v_{0} \in L_{\sigma}^{\infty} .
$$

Proof. We observe that

$$
\sup _{t \in \gamma}\left\|S(t) v_{0}\right\|_{L^{\infty}} \leq C\left\|v_{0}\right\|_{L^{\infty}}, \quad v_{0} \in D(A) \cap L^{2} .
$$

The estimate (5.9) holds for $|t| \leq 1$ by Proposition 3.3 (ii). For $|t| \geq 1$, we take $t_{1}=e^{i \theta}$ and set $t=t_{1}+t_{2}$. Since $S\left(t_{1}\right) v_{0} \in D(A) \cap L^{2}$, by (5.4) we estimate

$$
\left\|S(t) v_{0}\right\|_{L^{\infty}}=\left\|S\left(t_{2}\right) S\left(t_{1}\right) v_{0}\right\|_{L^{\infty}} \leq C\left\|S\left(t_{1}\right) v_{0}\right\|_{D(A)} \leq C^{\prime}\left\|v_{0}\right\|_{L^{\infty}} .
$$

Thus (5.9) holds. By (4.4), (5.9) and (5.2), we obtain 
$\sup \left\{\left\|S(t) v_{0}\right\|_{L^{\infty}} \mid 0 \leq \arg t \leq \theta\right\} \leq C\left\|v_{0}\right\|_{L^{\infty}}$.

Since the same estimate holds for $-\theta \leq \arg t \leq 0$, (5.8) holds for $v_{0} \in D(A) \cap L^{2}$.

For $v_{0} \in L_{\sigma}^{\infty}$ we take a sequence $\left\{v_{0, m}\right\} \subset C_{c, \sigma}^{\infty}$ satisfying (3.1). Then by choosing a subsequence (still denoted by $v_{m}$ ), $v_{m}=S(t) v_{0, m}$ converges to $S(t) v_{0}$ locally uniformly in $\bar{\Omega} \times(0, \infty)$ by Proposition 3.2 (ii). By (5.8) and (3.1), we have

$$
\sup _{t \in \Sigma_{\theta}}\left\|v_{m}\right\|_{L^{\infty}} \leq C\left\|v_{0}\right\|_{L^{\infty}} .
$$

Since $v_{m}$ is uniformly bounded in $\Omega \times \Sigma_{\theta}, \partial_{t}^{s} \partial_{x}^{k} v_{m}$ is uniformly bounded in $\Omega \times\{\delta \leq$ $\operatorname{Re} t,|\arg t| \leq \theta\}$ for $2 s+|k| \leq 2$ and each $\delta>0$. Thus by choosing a subsequence, $v_{m}$ converges to a limit $v$ locally uniformly in $\bar{\Omega} \times\{\delta \leq \operatorname{Re} t,|\arg t| \leq \theta\}$. Since $v_{m}(\cdot, t)$ satisfies (3.2), the limit $v(\cdot, t)$ is also analytic on $L^{\infty}$ for $t \in \Sigma_{\theta}$. Since $v$ agrees with $S(t) v_{0}$ for $t \in(0, \infty)$, we have $v=S(t) v_{0}$ for all $t \in \Sigma_{\theta}$. Since (5.10) is inherited to $v=S(t) v_{0},(5.8)$ holds. The proof is now complete.

Proof of Theorem 1.2. The assertion follows from Lemma 5.3.

Proof of Theorem 1.3. For $n=2$ and solutions with zero net force, the spatial decay estimates (3.19) and (3.20) hold. By using (3.19) and the Liouville theorem (Theorem 1.1) for $p \in(2, \infty)$, we are able to prove (4.4) in the same way as $n \geq 3$. For complex time, we use (3.20).

Remarks 5.4. (i) (Decay for the time derivative) Theorems 1.2 implies the large time estimate

$$
\sup _{t>0}\left\{\left\|S(t) v_{0}\right\|_{L^{\infty}}+t\left\|A S(t) v_{0}\right\|_{L^{\infty}}\right\} \leq C\left\|v_{0}\right\|_{L^{\infty}}, \quad v_{0} \in L_{\sigma}^{\infty} .
$$

Since $S(t)$ is bounded on $\gamma=\{\arg t=\theta\}$ for $\theta \in(0, \pi / 2)$, we are able to change the integral path of the Laplace transform from $(0, \infty)$ to $\gamma$, i.e.,

$$
(\lambda-A)^{-1} f=\int_{0}^{\infty} e^{-\lambda t} S(t) f \mathrm{~d} t=\int_{\gamma} e^{-\lambda t} S(t) f \mathrm{~d} t .
$$

See [50, Theorem 5.2]. This gives the resolvent estimate

$$
\left\|(\lambda-A)^{-1} f\right\|_{L^{\infty}} \leq \frac{C_{\theta}}{|\lambda|}\|f\|_{L^{\infty}}, \quad \lambda \in \Sigma_{\theta+\pi / 2}, \quad \theta \in(0, \pi / 2) .
$$


Since $S(t)$ is represented by the Dunford integral of the resolvent $(\lambda-A)^{-1},(5.11)$ follows from (5.12).

(ii) (Decay for the spatial derivative) By (3.4), for each $T>0$ we have

$$
\left\|\nabla S(t) v_{0}\right\|_{L^{\infty}} \leq \frac{C}{t^{1 / 2}}\left\|v_{0}\right\|_{L^{\infty}}, \quad 0<t \leq T .
$$

We are not able to take $T=\infty$ in (5.13). To see this, we recall the decay estimate

$$
\left\|\nabla S(t) v_{0}\right\|_{L^{p}} \leq \frac{C}{t^{n / 2(1 / q-1 / p)+1 / 2}}\left\|v_{0}\right\|_{L^{q}}, \quad t>0, v_{0} \in L_{\sigma}^{q}, 1<q \leq p \leq n .
$$

See [38] for $n \geq 3$, [24] for $n=2$ and [45]. The condition $p \leq n$ is optimal in the sense that (5.14) for $p>n$ is not valid for all $t \geq 1$ and $v_{0} \in L_{\sigma}^{q}$ [45], [37, Corollary 2.4]. If (5.13) were true for all $t>0$, by the semigroup property and the decay estimate

$$
\left\|S(t) v_{0}\right\|_{L^{\infty}} \leq \frac{C}{t^{n /(2 q)}}\left\|v_{0}\right\|_{L^{q}}, \quad t>0
$$

proved in [38], [23] for $n \geq 3$, [25] for $n=2$, we would obtain (5.14) for $p=\infty$.

(iii) Theorem 1.3 improves the pointwise estimates of the two-dimensional Navier-Stokes flows for rotationally symmetric initial data around a unit disk $\Omega^{c}$. Let $u$ be a global-intime solution of the Navier-Stokes equations for initial data $u_{0} \in L_{\sigma}^{2} \cap L^{1} \cap W_{0}^{2-2 / q, q}(\Omega)$ for $q \in(1,4 / 3]$. It is proved in [35, Theorem 5.8] that if $u_{0}$ is $D_{m+2}$-covariant for some $m \geq 0$ and satisfies $u_{0} \in W_{0}^{1,2}(\Omega),(1+|x|)^{m+3}\left|u_{0}(x)\right| \in L^{\infty}(\Omega)$ and $(1+|x|)^{m+1}\left|u_{0}(x)\right| \in L^{1}(\Omega)$, then $u$ is $D_{m+2}$-covariant and satisfies the pointwise estimates

$$
|u(x, t)| \lesssim \begin{cases}|x|^{-(m+3)}, & |x| \geq 2, t>0, \\ t^{-(m+3) / 2}, & x \in \Omega, t>0 .\end{cases}
$$

The estimate (5.15) is obtained from the representation formula of the Navier-Stokes flows. Although the right-hand side is unbounded at $x=0$ and $t=0$, respectively, by estimating the integral form of $u$ by using (1.6), we are able to show that $u$ is bounded in $\Omega \times(0, \infty)$. Hence (5.15) is improved to

$$
|u(x, t)| \lesssim\left\{\begin{array}{l}
(1+|x|)^{-(m+3)}, \\
(1+t)^{-(m+3) / 2}, \quad x \in \Omega, t>0,
\end{array}\right.
$$

as noted in [35, p.1546, Remarks (ii)]. 


\section{ACKNOWLEDGEMENTS}

The author is grateful to Professor Toshiaki Hishida for informing him of the paper [37] on the optimal exponent of the decay estimate (5.14). This work is partially supported by JSPS through the Grant-in-aid for Young Scientist (B) 17K14217, Scientific Research (B) 17H02853 and Osaka City University Advanced Mathematical Institute (MEXT Joint Usage / Research Center on Mathematics and Theoretical Physics).

\section{REFERENCES}

[1] K. Abe. The Stokes semigroup on non-decaying spaces. Ph.D. Thesis, The University of Tokyo, 2013.

[2] K. Abe. Global well-posedness of the two-dimensional exterior Navier-Stokes equations for non-decaying data. Arch. Rational Mech. Anal., 227:69-104, (2018).

[3] K. Abe and Y. Giga. Analyticity of the Stokes semigroup in spaces of bounded functions. Acta Math., 211:1-46, (2013).

[4] K. Abe and Y. Giga. The $L^{\infty}$-Stokes semigroup in exterior domains. J. Evol. Equ., 14:1-28, (2014).

[5] K. Abe, Y. Giga, and M. Hieber. Stokes resolvent estimates in spaces of bounded functions. Ann. Sci. Éc. Norm. Supér., 48:537-559, (2015).

[6] K. Abe, Y. Giga, K. Schade, and T. Suzuki. On the Stokes semigroup in some non-Helmholtz domains. Arch. Math., 104:177-187, (2015).

[7] K. Abe, Y. Giga, K. Schade, and T. Suzuki. On the Stokes resolvent estimates for cylindrical domains. J. Evol. Equ., 17:17-49, (2017).

[8] W. Arendt, C. J. K. Batty, M. Hieber, and F. Neubrander. Vector-valued Laplace transforms and Cauchy problems. Birkhäuser/Springer Basel AG, Basel, second edition, 2011.

[9] T. Barker and G. Seregin. Ancient solutions to Navier-Stokes equations in half space. J. Math. Fluid Mech., 17:551-575, (2015).

[10] M. E. Bogovskir. Solution of the first boundary value problem for an equation of continuity of an incompressible medium. Dokl. Akad. Nauk SSSR, 248:1037-1040, (1979).

[11] M. Bolkart. The Stokes Equations in Spaces of Bounded Functions and Spaces of Bounded Mean Oscillation. PhD Thesis, TU Darmstadt, 2016.

[12] M. Bolkart and M. Hieber. Pointwise upper bounds for the solution of the Stokes equation on $L_{\sigma}^{\infty}(\Omega)$ and applications. J. Funct. Anal., 268:1678-1710, (2015).

[13] W. Borchers and T. Miyakawa. $L^{2}$ decay for the Navier-Stokes flow in halfspaces. Math. Ann., 282:139$155,(1988)$.

[14] W. Borchers and T. Miyakawa. On some coercive estimates for the Stokes problem in unbounded domains. In The Navier-Stokes equations II-theory and numerical methods (Oberwolfach, 1991), volume 1530 of Lecture Notes in Math., pages 71-84. Springer, Berlin, 1992.

[15] W. Borchers and H. Sohr. On the semigroup of the Stokes operator for exterior domains in $L^{q}$-spaces. Math. Z., 196:415-425, (1987).

[16] W. Borchers and H. Sohr. On the equations $\operatorname{rot} \mathbf{v}=\mathbf{g}$ and $\operatorname{div} \mathbf{u}=f$ with zero boundary conditions. Hokkaido Math. J., 19:67-87, (1990).

[17] W. Borchers and W. Varnhorn. On the boundedness of the Stokes semigroup in two-dimensional exterior domains. Math. Z., 213:275-299, (1993).

[18] L. Brandolese. Space-time decay of Navier-Stokes flows invariant under rotations. Math. Ann., 329:685706, (2004).

[19] L. Cattabriga. Su un problema al contorno relativo al sistema di equazioni di Stokes. Rend. Sem. Mat. Univ. Padova, 31:308-340, (1961).

[20] D. Chae and J. Wolf. On Liouville type theorems for the steady Navier-Stokes equations in $\mathbb{R}^{3}$. J. Differential Equations, 261:5541-5560, (2016).

[21] D. Chae and T. Yoneda. On the Liouville theorem for the stationary Navier-Stokes equations in a critical space. J. Math. Anal. Appl., 405:706-710, (2013).

[22] I.-D. Chang and R. Finn. On the solutions of a class of equations occurring in continuum mechanics, with application to the Stokes paradox. Arch. Rational Mech. Anal., pages 388-401, (1961). 
[23] Z. M. Chen. Solutions of the stationary and nonstationary Navier-Stokes equations in exterior domains. Pacific J. Math., 159:227-240, (1993).

[24] W. Dan and Y. Shibata. On the $L_{q}-L_{r}$ estimates of the Stokes semigroup in a two-dimensional exterior domain. J. Math. Soc. Japan, 51:181-207, (1999).

[25] W. Dan and Y. Shibata. Remark on the $L_{q}-L_{\infty}$ estimate of the Stokes semigroup in a 2-dimensional exterior domain. Pacific J. Math., 189:223-239, (1999).

[26] E. B. Davies. Heat kernels and spectral theory, volume 92 of Cambridge Tracts in Mathematics. Cambridge University Press, Cambridge, 1989.

[27] W. Desch, M. Hieber, and J. Prüss. $L^{p}$-theory of the Stokes equation in a half space. J. Evol. Equ., 1:115$142,(2001)$.

[28] L. C. Evans. Partial differential equations, volume 19 of Graduate Studies in Mathematics. American Mathematical Society, Providence, RI, second edition, 2010.

[29] D. Fujiwara and H. Morimoto. An $L_{r}$-theorem of the Helmholtz decomposition of vector fields. J. Fac. Sci. Univ. Tokyo Sect. IA Math., 24:685-700, (1977).

[30] G. P. Galdi. An introduction to the mathematical theory of the Navier-Stokes equations. Springer Monographs in Mathematics. Springer, New York, second edition, 2011.

[31] Y. Giga. Analyticity of the semigroup generated by the Stokes operator in $L_{r}$ spaces. Math. Z., 178:297329, (1981).

[32] Y. Giga, P.-Y. Hsu, and Y. Maekawa. A Liouville theorem for the planer Navier-Stokes equations with the no-slip boundary condition and its application to a geometric regularity criterion. Comm. Partial Differential Equations, 39:1906-1935, (2014).

[33] Y. Giga and H. Sohr. On the Stokes operator in exterior domains. J. Fac. Sci. Univ. Tokyo Sect. IA Math., 36:103-130, (1989).

[34] L. C. Grove and C. T. Benson. Finite reflection groups, volume 99 of Graduate Texts in Mathematics. Springer-Verlag, New York, second edition, 1985.

[35] C. He and T. Miyakawa. Nonstationary Navier-Stokes flows in a two-dimensional exterior domain with rotational symmetries. Indiana Univ. Math. J., 55:1483-1555, (2006).

[36] M. Hieber and P. Maremonti. Bounded analyticity of the Stokes semigroup on spaces of bounded functions. In Recent developments of mathematical fluid mechanics, Adv. Math. Fluid Mech., pages 275-289. Birkhäuser/Springer, Basel, 2016.

[37] T. Hishida. On the relation between the large time behavior of the Stokes semigroup and the decay of steady Stokes flow at infinity. In Parabolic problems, volume 80 of Progr. Nonlinear Differential Equations Appl., pages 343-355. Birkhäuser/Springer Basel AG, Basel, (2011).

[38] H. Iwashita. $L_{q}-L_{r}$ estimates for solutions of the nonstationary Stokes equations in an exterior domain and the Navier-Stokes initial value problems in $L_{q}$ spaces. Math. Ann., 285:265-288, (1989).

[39] H. Jia, G. Seregin, and V. Šverák. Liouville theorems in unbounded domains for the time-dependent Stokes system. J. Math. Phys., 53:115604, 9, (2012).

[40] G. Koch, N. Nadirashvili, G. A. Seregin, and V. Šverák. Liouville theorems for the Navier-Stokes equations and applications. Acta Math., 203:83-105, (2009).

[41] H. Kozono, Y. Terasawa, and Y. Wakasugi. A remark on Liouville-type theorems for the stationary NavierStokes equations in three space dimensions. J. Funct. Anal., 272:804-818, (2017).

[42] A. Lunardi. Analytic semigroups and optimal regularity in parabolic problems. Progress in Nonlinear Differential Equations and their Applications, 16. Birkhäuser Verlag, Basel, 1995.

[43] P. Maremonti. On the Stokes problem in exterior domains: the maximum modulus theorems. Discrete Contin. Dyn. Syst., 34:2135-2171, (2014).

[44] P. Maremonti and S. Shimizu. Global existence of solutions to 2-D Navier-Stokes flow with non-decaying initial data in exterior domains. J. Math. Fluid Mech., 20:899-927, (2018).

[45] P. Maremonti and V. A. Solonnikov. On nonstationary Stokes problem in exterior domains. Ann. Scuola Norm. Sup. Pisa Cl. Sci. (4), 24:395-449, (1997).

[46] K. Masuda. On the generation of analytic semigroups of higher-order elliptic operators in spaces of continuous functions (in japanese). Proc. Katata Symposium on Partial Differential Equations, (1972).

[47] M McCracken. The resolvent problem for the Stokes equations on halfspace in $L_{p}$. SIAM J. Math. Anal., 12:201-228, (1981). 
[48] T. Miyakawa. On nonstationary solutions of the Navier-Stokes equations in an exterior domain. Hiroshima Math. J., 12:115-140, (1982).

[49] R. Mizumachi. On the asymptotic behavior of incompressible viscous fluid motions past bodies. J. Math. Soc. Japan, 36:497-522, (1984).

[50] A. Pazy. Semigroups of linear operators and applications to partial differential equations. Springer-Verlag, New York, 1983.

[51] J. Saal. The Stokes operator with Robin boundary conditions in solenoidal subspaces of $L^{1}\left(\mathbb{R}_{+}^{n}\right)$ and $L^{\infty}\left(\mathbb{R}_{+}^{n}\right)$. Comm. Partial Differential Equations, 32:343-373, (2007).

[52] G. Seregin. Liouville theorem for 2D Navier-Stokes equations in a half space. J. Math. Sci. (N.Y.), 210:849856, (2015).

[53] G. Seregin. Liouville type theorem for stationary Navier-Stokes equations. Nonlinearity, 29:2191-2195, (2016).

[54] G. Seregin and V. Šverák. On type I singularities of the local axi-symmetric solutions of the Navier-Stokes equations. Comm. Partial Differential Equations, 34:171-201, (2009).

[55] G. Seregin and W. Wang. Sufficient conditions on Liouville type theorems for the 3D steady Navier-Stokes equations. Algebra i Analiz, 31:269-278, (2019).

[56] C. G. Simader and H. Sohr. A new approach to the Helmholtz decomposition and the Neumann problem in $L^{q}$-spaces for bounded and exterior domains. In Mathematical problems relating to the Navier-Stokes equation, volume 11 of Ser. Adv. Math. Appl. Sci., pages 1-35. World Sci. Publ., River Edge, NJ, 1992.

[57] V. A. Solonnikov. Estimates of the solution of a certain initial-boundary value problem for a linear nonstationary system of Navier-Stokes equations. Zap. Naučn. Sem. Leningrad. Otdel Mat. Inst. Steklov. (LOMI), 59:178-254, 257, (1976). Boundary value problems of mathematical physics and related questions in the theory of functions, 9.

[58] V. A. Solonnikov. On nonstationary Stokes problem and Navier-Stokes problem in a half-space with initial data nondecreasing at infinity. J. Math. Sci. (N. Y.), 114:1726-1740, (2003).

[59] V. A. Solonnikov. Weighted Schauder estimates for evolution Stokes problem. Ann. Univ. Ferrara Sez. VII Sci. Mat., 52:137-172, (2006).

[60] V. A. Solonnikov. Schauder estimates for the evolutionary generalized Stokes problem. In Nonlinear equations and spectral theory, volume 220 of Amer. Math. Soc. Transl. Ser. 2, pages 165-200. Amer. Math. Soc., Providence, RI, 2007.

[61] H. B. Stewart. Generation of analytic semigroups by strongly elliptic operators. Trans. Amer. Math. Soc., 199:141-162, (1974).

[62] S. Ukai. A solution formula for the Stokes equation in $\mathbf{R}_{+}^{n}$. Comm. Pure Appl. Math., 40:611-621, (1987).

[63] W. Varnhorn. The boundary value problems of the Stokes resolvent equations in $n$ dimensions. Math. Nachr., 269/270:210-230, (2004).

[64] L. von Below and M. Bolkart. The Stokes equations in layer domains on spaces of bounded and integrable functions. Math. Nachr., 290:1553-1587, (2017).

(K. ABE) Department of Mathematics, Graduate School of Science, Osaka City University, 3-3-138 SugIMOTO, SUMIYoshi-Ku OSAKA, 558-8585, JAPAN

E-mail address: kabe@sci.osaka-cu.ac.jp 